\title{
Numerical investigations of parametric decay into trapped waves in magnetized plasmas with a non-monotonic density background
}

\author{
Senstius, M.G.; Nielsen, S.K.; Vann, R.G.L.
}

Published in:

Physics of Plasmas

Link to article, DOI:

$10.1063 / 5.0004672$

Publication date:

2020

Document Version

Publisher's PDF, also known as Version of record

Link back to DTU Orbit

Citation (APA):

Senstius, M. G., Nielsen, S. K., \& Vann, R. G. L. (2020). Numerical investigations of parametric decay into trapped waves in magnetized plasmas with a non-monotonic density background. Physics of Plasmas, 27, [062102 ]. https://doi.org/10.1063/5.0004672

\section{General rights}

Copyright and moral rights for the publications made accessible in the public portal are retained by the authors and/or other copyright owners and it is a condition of accessing publications that users recognise and abide by the legal requirements associated with these rights.

- Users may download and print one copy of any publication from the public portal for the purpose of private study or research.

- You may not further distribute the material or use it for any profit-making activity or commercial gain

- You may freely distribute the URL identifying the publication in the public portal 


\section{Numerical investigations of parametric decay into trapped waves in magnetized plasmas with a non-monotonic density background $\odot$}

Cite as: Phys. Plasmas 27, 062102 (2020); https://doi.org/10.1063/5.0004672

Submitted: 18 February 2020 . Accepted: 13 May 2020 . Published Online: 05 June 2020

(D) M. G. Senstius, (iD) S. K. Nielsen, and (iD) R. G. L. Vann

COLLECTIONS

F This paper was selected as Featured
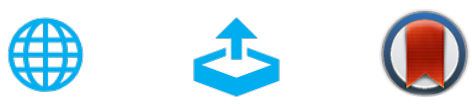

View Online

Export Citation

\section{ARTICLES YOU MAY BE INTERESTED IN}

Gyrokinetic understanding of the edge pedestal transport driven by resonant magnetic perturbations in a realistic divertor geometry

Physics of Plasmas 27, 062301 (2020); https://doi.org/10.1063/1.5144445

Role of sheared $\mathrm{E} \times \mathrm{B}$ flow in self-organized, improved confinement states in magnetized plasmas

Physics of Plasmas 27, 060501 (2020); https://doi.org/10.1063/1.5142734

Magnetohydrodynamic Richtmyer-Meshkov instability under an arbitrarily oriented magnetic field

Physics of Plasmas 27, 062101 (2020); https://doi.org/10.1063/1.5142042

\section{AlP Advances Fluids and Plasmas Collection}




\title{
Numerical investigations of parametric decay into trapped waves in magnetized plasmas with a non-monotonic density background $\odot$
}

Cite as: Phys. Plasmas 27, 062102 (2020); doi: 10.1063/5.0004672

Submitted: 18 February 2020 - Accepted: 13 May 2020 .

Published Online: 5 June 2020

M. G. Senstius, ${ }^{1, a)}$ (D) S. K. Nielsen, ${ }^{1}$ (D) and R. G. L. Vann ${ }^{2}$ (iD

\begin{abstract}
AFFILIATIONS
'Department of Physics, Technical University of Denmark, Fysikvej, DK-2800 Kgs. Lyngby, Denmark

${ }^{2}$ York Plasma Institute, Department of Physics, University of York, York YO1O 5DD, United Kingdom
\end{abstract}

a) Author to whom correspondence should be addressed: mgse@fysik.dtu.dk

\begin{abstract}
Parametric decay instabilities (PDIs) exciting daughter waves trapped inside a magnetized plasma with a non-monotonic density profile are investigated numerically. The investigation is motivated in particular by observations of low threshold PDI signatures during second harmonic electron cyclotron resonance heating experiments in magnetically confined fusion experiments. We use the particle-in-cell code $\mathrm{EPOCH}$ to study conversion of a fast X-mode pump wave into a combination of half frequency X-mode and electron Bernstein waves and identify two regimes where PDIs can excite trapped electrostatic waves. Above the second harmonic upper hybrid (UH) density, a PDI known also as a two plasmon decay (TPD) instability excites a pair of UH waves that we locate in frequency and wavenumber space. At lower densities, a PDI known as stimulated Raman scattering may produce one trapped and one returning X-mode daughter wave with a much slower growth rate than the TPD instability. In both cases, we show that the frequency separation of the daughter waves depends on the density in a predictable manner. With little loss from the decay region, the trapped daughter waves become unstable with respect to secondary parametric instabilities (PIs), leading to distinctly different phases of the UH spectrum. Unlike the primary instability, the secondary PIs are shown to depend on ion dynamics. Furthermore, we observe escaping waves near the $3 / 2$ pump frequency resulting from tertiary PIs in agreement with recently proposed backscattering during magnetically confined fusion experiments.
\end{abstract}

Published under license by AIP Publishing. https://doi.org/10.1063/5.0004672

\section{INTRODUCTION}

Parametric instabilities (PIs) are nonlinear wave interaction instabilities whose three-wave interactions form a notable subgroup. These wave interactions occur when a strong pump wave is sent through a nonlinear medium such as a plasma, a fluid, ${ }^{1,2}$ or a nonlinear optical crystal. ${ }^{3}$ The pump wave may then decay into or combine with natural modes of the medium to produce other waves. A decay instability is typically referred to as a parametric decay instability (PDI). PDIs obey conservation of frequency and wave vector, leading to selection rules that, in an inhomogeneous medium, may only be satisfied locally. Early work on PDIs ${ }^{4-9}$ in plasmas showed that the pump wave amplitude threshold in an inhomogeneous plasma could be significantly higher than in a homogeneous plasma due to convection of the daughter waves out of the decay region.

Signatures of PDIs are typically observed in plasmas as emission patterns stimulated by externally launched electromagnetic waves. Such stimulated emission spectra have been observed directly in studies of the ionosphere since the early $1980 \mathrm{~s}^{10}$ where high power radio waves were launched from the ground into the ionosphere and the obtained scattering spectra contained peaks shifted from the injected frequency by natural frequencies of the plasma. Since the waves involved in PDIs depend on the plasma parameters in the decay region, the scattering spectra produced by PDIs can be used to diagnose the ionosphere. ${ }^{11}$ Waves injected into the ionosphere or a laboratory plasma may not produce the observed signatures of PDI directly. Instead, the injected waves might have to undergo several conversion processes before the observed emission spectra appear. PDIs are more likely to occur in certain parts of the plasma, such as the resonant region known as the upper hybrid (UH) layer. Near the UH layer, both X-mode and electron Bernstein waves (EBWs) experience large changes in their wave vectors and wave amplification, ${ }^{12}$ which can facilitate PDIs at reduced pump power thresholds. Unlike X-mode waves, EBWs cannot be injected into a plasma from vacuum but are typically excited through linear conversion of X-mode at the UH layer 
in $\mathrm{O}-\mathrm{X}-\mathrm{B}^{13}$ and $\mathrm{X}-\mathrm{B}^{14}$ conversion schemes. Amplification in the conversion region can lead to PDIs, which have been observed in low temperature laboratory plasmas, ${ }^{15}$ and the escaping signatures of the PDIs can be taken as an indication that a strong X-mode wave has made it to the UH layer and that linear conversion into EBWs therefore also has occurred. ${ }^{16}$ In magnetically confined fusion plasmas, signatures of PDIs near the UH layer were first observed in the Versator II tokamak during electron cyclotron resonance heating (ECRH). ${ }^{17}$ Later, observations have been made at a number of devices including during ECRH and EBW heating at the Wendelstein $7-\mathrm{AS}^{18,19}$ stellarator, the FT- $1^{20}$ tokamak, the TCA ${ }^{21}$ tokamak, the MAST ${ }^{22}$ tokamak, the TJ-K ${ }^{23}$ stellarator, and the $\mathrm{LHD}^{24}$ stellarator and during collective Thomson scattering (CTS ${ }^{25-27}$ at the ASDEX Upgrade tokamak. PDI has also been studied experimentally in other magnetically confined laboratory plasmas such as in tandem mirror machines ${ }^{28}$ and linear devices. ${ }^{29,3}$

Although wave amplification of X-mode waves at the UH layer may lead to PDIs at a reduced power threshold, the amplification is not a necessary condition for the nonlinear wave interactions to become unstable, and there are other effects that may lower the PDI power threshold. In ionospheric heating experiments, electromagnetic waves launched from the ground can give rise to density striation and excite waves that are trapped inside them. Because there is little convection out of the trapping region, the trapped waves may become unstable to cascades of PDIs, which produce new waves escaping back to Earth where they are observed in rich frequency spectra. ${ }^{31,32}$ The buildup of large amplitude electrostatic waves in striations has been studied numerically using Vlasov solvers and has been proposed as a means of plasma heating. ${ }^{33,34}$ In inertial confinement fusion, the high power lasers used to compress fuel pellets may become unstable to a PDI known as a two plasmon decay (TPD) instability ${ }^{35,36}$ near the quarter critical surface where half the laser pump frequency corresponds to the plasma frequency. Because the quarter critical surface is close to the second harmonic UH layer, the injected wave experiences no significant amplification and is not trapped, but the process still becomes unstable due to the high intensity of the lasers. This PDI was also observed experimentally in the $1980 \mathrm{~s}^{37}$ and leads to strong electron heating, which in turn deteriorates pellet detonation significantly. Signatures of PDIs have also been observed in tokamaks during second harmonic ECRH where the fundamental UH layer typically is not found inside the confined plasma, and the gyrotron beam, therefore, is not significantly amplified anywhere. Although modern tokamaks and stellarators have installed multiple gyrotrons for heating, current drive, and diagnostics, each producing $1 \mathrm{MW}$ beams, traditional estimates for PDI power thresholds during second harmonic ECRH suggest a PDI power threshold on the order of $1 \mathrm{GW}$ and thus far exceed what the gyrotrons are capable of delivering. ${ }^{38-41}$ Still, strong scattering was observed during second harmonic ECRH on the TEXTOR toka$\mathrm{mak}^{42-44}$ at a much lower gyrotron power of $\sim 100 \mathrm{~kW}$. The scattering was observed in the presence of a magnetic island and showed modulation at a frequency matching the island rotation. Similar observations have been made on ASDEX Upgrade ${ }^{45,46}$ in the presence of islands and edge localized modes. This has led to the idea that there is a mechanism that lowers the PDI power threshold in a non-monotonic density profile caused by such structures.

The PDI signatures observed at TEXTOR were peaks shifted in frequency from the gyrotron line by approximately an integer times the lower hybrid ( $\mathrm{LH})$ frequency. It was first proposed that the non- monotonic plasma density perturbation caused by the rotating island would allow for ion Bernstein waves (IBWs) near the LH frequency to be trapped inside the island. Theoretical estimates showed that a PDI of the gyrotron into an IBW and a downshifted backscattered wave would have a significantly reduced power threshold ${ }^{41,47}$ whenever the gyrotron passed through the island. However, the model showed slightly different frequency shifts than what was observed experimentally. Later, more elaborate models were proposed ${ }^{48-50}$ involving an initial TPD instability into two trapped half frequency UH waves, which further decay through cascades of secondary PDIs into an IBW and an increasingly downshifted UH wave. Secondary daughter waves could then build up and combine into escaping waves in tertiary PIs, bringing better agreement with the experimental observations to the theoretical models. The models suggest a number of complications to gyrotron operation in non-monotonic plasma density profiles such as a substantial unintentional power deposition into the islands ${ }^{29,52}$ and excitation of potentially damaging escaping waves at different halfinteger harmonics of the gyrotron frequency. ${ }^{45,46,56}$ The nonmonotonic density profile can be caused by a number of mechanisms, which means that such cascades of PDIs might be common during gyrotron operation across many machines without being noticed simply because no diagnostic is looking for them.

In this article, we use the particle-in-cell (PIC) code $\mathrm{EPOCH}^{53,54}$ to study PDIs into one or two trapped UH waves due to a nonmonotonic plasma density profile. Using X-mode and EBW dispersion relations, we investigate two processes, TPD and stimulated Raman scattering, which may produce the trapped waves from an X-mode pump wave. This is the necessary first step of the models explaining the PDI signatures during second harmonic ECRH at tokamaks such as TEXTOR and ASDEX Upgrade. We show that trapped daughter waves of such PDIs can be excited numerically and locate them in frequency and wavenumber space. Examining spectra for different density profiles, we investigate how the frequency of the primary daughter waves depends on them and discuss growth rates for different instabilities. We compare simulations with mobile and immobile ions to confirm that the primary PDIs are independent of ion waves but that the secondary PDIs are not. We look into and confirm numerically that a backscattered $3 / 2 f_{0}$ wave is generated due to the plasma profile and the trapped waves within it. Finally, we compare 1D and 2D simulations in terms of their growth rate and show that wave structures, which cannot be excited in 1D, appear in 2D.

\section{THEORY OF TPD AND PIC CODES}

PDIs can occur whenever a strong pump wave propagates through a medium that has a second order nonlinearity. Conservation of energy and momentum of the waves can be expressed as conservation of frequency, $f$, and wave vector, $\mathbf{k}$, leading to the selection rules for the daughter waves,

$$
f_{0}=f_{1}+f_{2}, \quad \mathbf{k}_{0}=\mathbf{k}_{1}+\mathbf{k}_{2},
$$

where $f_{j}$ and $\mathbf{k}_{j}$ are the frequency and wave vector of the $\mathrm{jth}$ wave (see Fig. 1). Index 0 refers to the pump wave, while 1 and 2 refer to the daughter waves, which for the TPD instability will be referred to as the primary daughter waves. The growth rates of the daughter waves depend on the amplitude of the pump wave, and the parametric decay becomes unstable if the growth rates exceed the losses of the daughter waves in the decay region where the selection rules are satisfied. This 


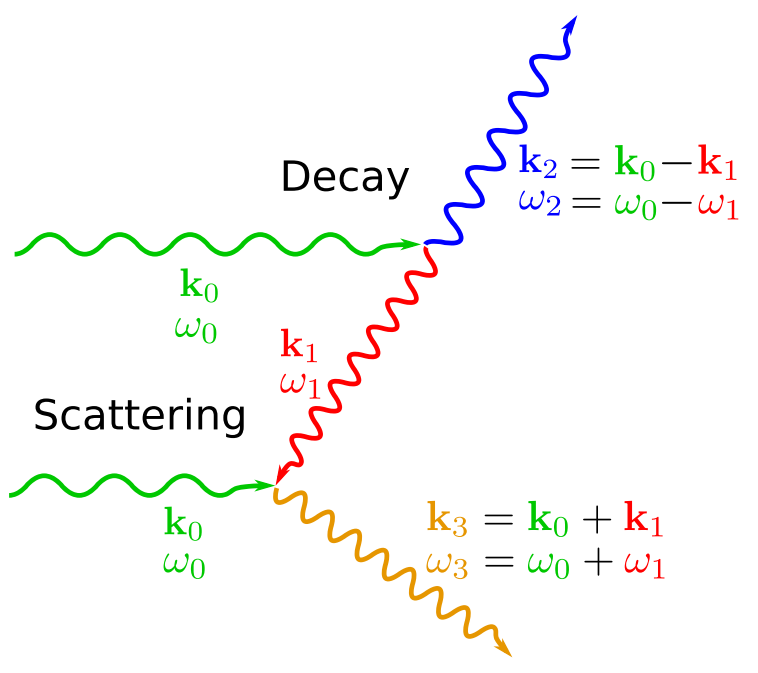

FIG. 1. An illustration of two types of Pls involving three waves: a decay into two waves and scattering or combination of two waves. Both satisfy Eq. (1). As indicated, a long wavelength wave may decay into two short wavelength waves provided that their $k$-vectors point in different directions, which would be the typical situation for TPD.

means that decay into waves other than natural modes, which are heavily dampened, is unlikely to occur. In an inhomogeneous plasma, convection of the daughter waves out of the decay region can lead to significant losses, and PDIs may then only become convectively unstable, simply amplifying the daughter waves as they pass through the decay region. Decay into waves that are somehow not lost from the decay region, on the other hand, has a lowered pump amplitude threshold, and the instabilities may become absolute such that the daughter waves grow exponentially in time. Although trapped waves may experience reduced losses from convection, they can still be dampened due to, e.g., collisions and diffraction perpendicular to wave propagation.

In a plasma, electromagnetic and electrostatic waves cause mobile charges, i.e., electrons and ions, to be accelerated by the Lorentz force, generating a current. For large wave amplitudes, the induced current is not linear in the wave amplitude, which can facilitate nonlinear wave interactions such as PDIs. A large amplitude ECRH beam will pass the second harmonic UH layer before it reaches the second harmonic electron cyclotron resonance. Here, thermally excited half frequency $\mathrm{UH}$ waves, more specifically slow X-mode and EBWs, may interact with the gyrotron beam if the selection rules in (1) are satisfied. In a monotonic density region, the primary daughter waves are converted and reflected at the UH layer but are free to propagate toward the plasma core. In a non-monotonic density profile caused by an island, multiple UH layers may trap the primary daughter waves in the poloidal direction as displayed in Fig. 2. The combination of X-mode and EBWs that will satisfy the conservation rules will depend on local parameters, but in general, $\mathbf{k}_{1}$ and $\mathbf{k}_{2}$ will be of opposite sign in order to add up to that of the fast X-mode pump wave, which has a comparably low wave number. The primary daughter waves need not be exactly at half the frequency (a small mismatch in the frequencies is expected), but they must add up to that of the pump. As EBWs do not propagate below the electron cyclotron frequency, this is the lower bound for daughter

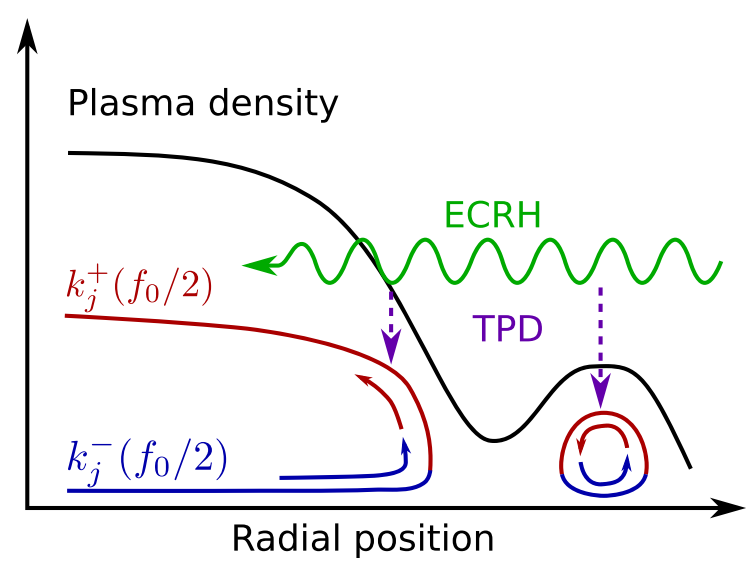

FIG. 2. A sketch showing how a non-monotonic density profile caused by a magnetic island can allow for UH waves to become trapped locally. An ECRH gyrotron beam, aiming for the plasma core, may decay through TPD near the second harmonic UH layer and excite two trapped half frequency waves inside the island. Linear conversion between X-mode and EBWs at the UH layer is the trapping mechanism. In the plasma core, waves can be convected away from the decay region.

wave frequencies. As the amplitudes of trapped waves build up, the waves can become unstable to secondary PDIs involving LH and IBWs that drain the primary daughter waves and thereby saturate the primary instability. Without ion dynamics, the secondary PDIs cannot saturate the primary TPD instability. Finally, the secondary daughter waves recombine in tertiary PIs to produce the escaping waves shifted in frequency, which were observed during experiments; see Fig. 3 for an overview of the cascade of PIs. In theory, it is also possible for the primary daughter waves to recombine directly with the pump wave to produce escaping fast X-mode waves near $3 f_{0} / 2$.

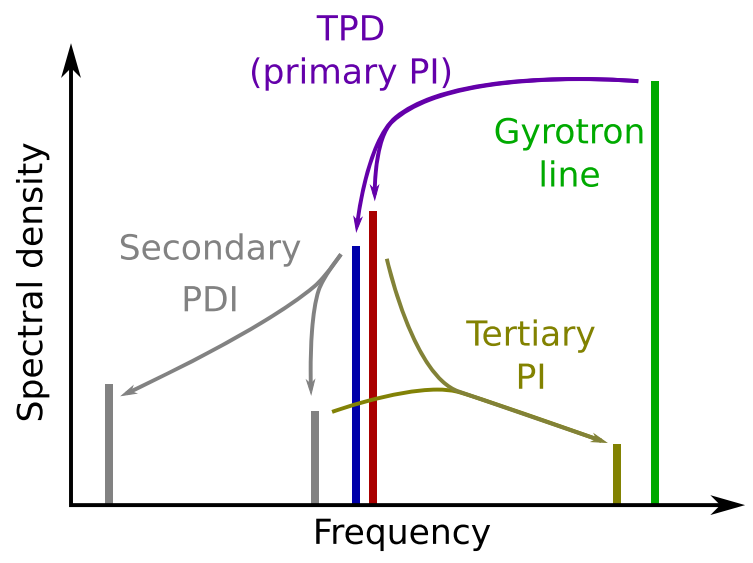

FIG. 3. A sketch of a parametric decay cascade, which is initiated by TPD of a gyrotron pump wave. First, two trapped half frequency UH waves are excited through PDI; these are the primary daughter waves. The primary daughter waves may decay further through secondary PDIs involving both $\mathrm{UH}$ and $\mathrm{LH}$ waves. Finally, tertiary PIs may combine excited trapped UH waves into escaping waves, shifted from the gyrotron in frequency. 
For the pump wave, a cold X-mode description will suffice far from the fundamental resonances. The dispersion relation is given by $^{50}$

$$
k_{0}=\frac{2 \pi f_{0}}{c} \sqrt{1-\frac{f_{\mathrm{pe}}^{2}}{f_{0}^{2}} \frac{f_{0}^{2}-f_{\mathrm{pe}}^{2}}{f_{0}^{2}-f_{\mathrm{UH}}^{2}},}
$$

where $k_{0}$ and $f_{0}$ are the pump wavenumber and frequency, respectively; $f_{\mathrm{UH}}^{2}=f_{\mathrm{pe}}^{2}+f_{\mathrm{ce}}^{2}$ is the $\mathrm{UH}$ frequency; and $f_{\mathrm{p} \sigma}^{2}$ $=q_{\sigma}^{2} n_{\sigma} /\left(4 \pi^{2} \varepsilon_{0} m_{\sigma}\right)$ and $f_{\mathrm{c} \sigma}=q_{\sigma} B_{0} /\left(2 \pi m_{\sigma}\right)$ are the plasma and cyclotron frequencies for species $\sigma$; above species, e refers to electrons, $q_{\sigma}, n_{\sigma}$, and $m_{\sigma}$ are the charge, density, and mass of species $\sigma$, respectively; $c$ is the speed of light; $\varepsilon_{0}$ is the vacuum permittivity; and $B_{0}$ is the background magnetic field. The primary daughter waves are either fast X-mode or EBWs, both satisfying the dispersion relation ${ }^{50,51}$

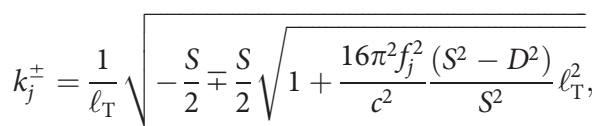

where $\ell_{\mathrm{T}}^{2}=3 v_{\mathrm{Te}}^{2} f_{\mathrm{pe}}^{2} /\left(8 \pi^{2}\left[4 f_{\mathrm{ce}}^{2}-f_{j}^{2}\right]\left[f_{j}^{2}-f_{\mathrm{ce}}^{2}\right]\right)$ with the electron thermal velocity defined as $v_{\mathrm{Te}}^{2}=2 T_{\mathrm{e}} / \mathrm{m}_{\mathrm{e}}$ from the electron temperature $T_{\mathrm{e}}$ and $S=1-f_{\mathrm{pe}}^{2} /\left(f_{j}^{2}-f_{\mathrm{ce}}^{2}\right)$ and $D=f_{\mathrm{ce}} f_{\mathrm{pe}}^{2} /\left[f_{j}\left(f_{j}^{2}-f_{\mathrm{ce}}^{2}\right)\right]$ being the sum and difference cold dielectric tensor elements as they are called in Stix notation. In the above equation, + refers to an EBW, while refers to X-mode. Both coincide at the $\mathrm{UH}$ layer, and the X-mode solution becomes the cold X-mode solution when moving away from the UH layer. Typically, $k_{j}^{ \pm}$is real on one side of the UH layer and complex on the other, and as X-mode waves are forward propagating waves and EBWs are backward propagating waves for underdense and moderately overdense plasmas, one wave incident on the UH layer will convert into the other and reflect.

While the present article considers magnetically confined fusion plasmas, TPD may also produce daughter waves in an unmagnetized plasma such as in inertial confinement fusion. This would instead produce a pair of Langmuir waves when $0.2 \leq f_{\mathrm{pe}}^{2} / f_{0}^{2} \leq 0.25$. However, the wave vector of the Langmuir daughter waves have nonzero components perpendicular to that of the pump ${ }^{36}$ and therefore need at least two spatial dimensions. TPD into X-mode and EBWs in a magnetized plasma does not require two spatial dimensions, which means that it is possible to investigate the mechanism in $1 \mathrm{D}$.

The PIC code we use in this article is EPOCH. ${ }^{53}$ It uses a second order split step routine, updating the electric and magnetic fields using discretized Maxwell's equations over a specified grid and propagating the particle positions and momenta with a relativistic particle pusher. To cut down on the computational demands of pushing every single particle around, particles are represented by a smaller number of clouds of real particles, also known as pseudo- or superparticles. The clouds are modeled using triangular distribution functions. EPOCH is capable of simulations featuring 1 to 3 spatial dimensions. Regardless of the number of spatial dimensions, all fields have three components. The remaining spatial dimensions are assumed homogeneous. PDIs have previously been observed in EPOCH simulations of EBW conversion schemes ${ }^{55}$ and of CTS experiments. ${ }^{54}$

\section{EXCITATION OF TRAPPED DAUGHTER WAVES}

Using $\mathrm{EPOCH}, 1 \mathrm{D}$ simulations are devised to investigate the excitation of trapped primary daughter waves of a TPD instability in a deuterium plasma with a non-monotonic density profile. The plasma consists of two species, e for electrons and i for deuterons, both assumed to be at a temperature of $T_{\mathrm{e}}=T_{\mathrm{i}}=100 \mathrm{eV}$. We have chosen deuterium, a typical ion species in a magnetically confined fusion plasma such as ASDEX Upgrade, but the choice of ion species is not expected to affect the excitation of primary daughter waves. The ion species affects mainly the secondary PDIs, which saturates the primary PDI. The EBWs and IBWs have very high wave numbers for low temperatures, so the temperatures are chosen to lower the wavenumbers while being in a range relevant to experiments. Electron and ion temperatures need not be equal. The magnetic field is assumed to be constant over the entire domain and pointing perpendicular to the direction of inhomogeneity, $B=B_{\mathrm{y}}=2.4 \mathrm{~T}$. A fast X-mode wave is sent from vacuum through a symmetric density bump and out into vacuum again along the $x$-axis. Inside the density bump, parameters are chosen such that the pump passes the second harmonic UH layer twice, and half frequency UH waves excited through TPD are trapped. We choose the frequency of the injected fast X-mode pump wave to be the common gyrotron frequency $f_{0}=140 \mathrm{GHz}$ used in machines such as TEXTOR, ASDEX Upgrade, and Wendelstein 7-X. The primary daughter waves might not both be exactly $f_{0} / 2=70 \mathrm{GHz}$, so combining Eqs. (1)-(3), i.e., the selection rules and dispersion relations, and allowing for a daughter frequency mismatch, the possible decays are shown in Fig. 4. This figure shows that a continuum of Xmode and EBWs around $70 \mathrm{GHz}$ may be excited by TPD. The aim of this article is to validate that decay into trapped waves occur in agreement with the theory, that is, distilled into Fig. 4.

The density profile for the first simulation is chosen to be

$$
n(x)= \begin{cases}n_{0}, & |x| \leq \delta, \\ n_{0}\left(1-\frac{|x|-\delta}{\ell}\right), & \delta \leq|x| \leq \delta+\ell, \\ 0, & \delta+\ell \leq|x| \leq w\end{cases}
$$

where $n_{\mathrm{e}}(x)=n_{\mathrm{i}}(x)=n(x), n_{0}=5.1 \times 10^{18} \mathrm{~m}^{-3}$ is the maximum density inside a homogeneous region of width $2 \delta=10 \mathrm{~mm}$ at the center, $\ell=4 \mathrm{~mm}$ is the gradient length on either side of the domain over

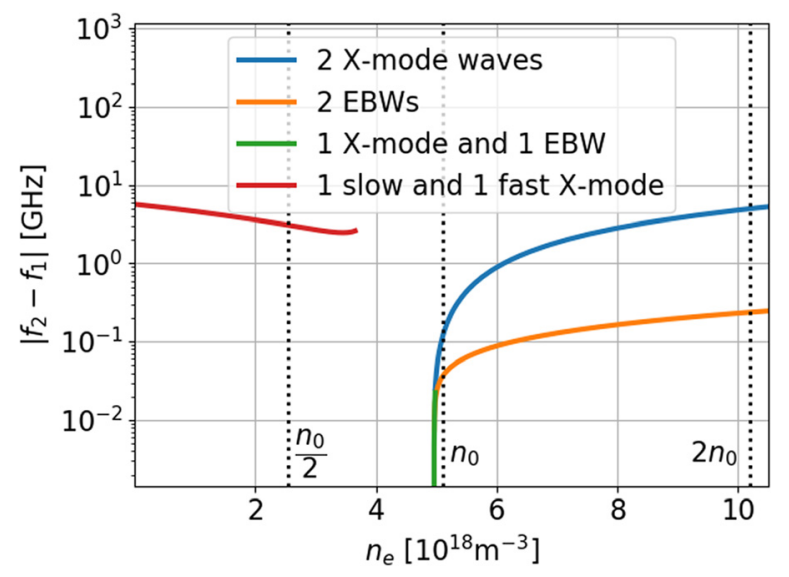

FIG. 4. Frequency mismatch of the primary daughter waves that satisfy the selection rules in Eq. (1) against density for $B=2.4 \mathrm{~T}$ and $T_{e}=100 \mathrm{eV}$. Colored solid lines refer to different types of primary decay daughter waves. Dotted lines mark peak densities in simulations that are run in this article. 
which the density drops linearly from $n_{0}$ to vacuum, and $2 w=20$ $\mathrm{mm}$ is the total width of the numerical domain. A $1 \mathrm{MW}$ Gaussian gyrotron beam of width $20 \mathrm{~mm}$ corresponds to an intensity of $I_{\text {pump }} \sim 1 \mathrm{~kW} / \mathrm{mm}^{2}$ around the center of the beam, which is a more convenient quantity to work with in $1 \mathrm{D}$ simulations. The pump is excited as an X-mode wave at the left $x=-10 \mathrm{~mm}$ boundary and is allowed to flow out of the right $x=10 \mathrm{~mm}$ boundary. The pump wave amplitude is ramped up using the amplitude envelope function $\frac{2}{\pi} \arctan \left(\frac{t}{\tau_{r}}\right)$, where $\tau_{r}=0.2 \mathrm{~ns}$. Particles leaving through the boundary are replaced by identical thermal particles entering the same boundary. This setup is run with $n_{\mathrm{x}}=1600$ grid points and a total of $n_{\text {part }}=3 \times 10^{4} n_{\mathrm{x}}$ particles. The number of grid points is chosen such that there are 20 points per wavelength of a wave of $k \approx 25 \mathrm{~mm}^{-1}$.

Figure 5 shows the density profile along with the wave number for UH waves at half the pump frequency. As is required for wave trapping, the X-mode and EBW lines form a closed loop, meeting at the UH layers, which means that a forward propagating X-mode wave reaching one end of the loop will be converted linearly into a backward propagating EBW and vice versa, preventing either from escaping. Due to the homogeneous center region, waves that satisfy the selection rules for $n_{e}=5.1 \times 10^{18} \mathrm{~m}^{-3}$ are expected to have the highest growth rate as they are found in their decay region for longer. This means that a pair of EBWs, $0.04 \mathrm{GHz}$ apart, and a pair of X-mode waves, $0.11 \mathrm{GHz}$ apart, will be dominating the $k$ - and $f$-power spectra near $70 \mathrm{GHz}$.

With the simulation parameters described above and a pump intensity of $I_{\text {pump }}=1 \mathrm{~kW} / \mathrm{mm}^{2}, \mathrm{EPOCH}$ is run. While EBWs are almost entirely longitudinal, the trapped slow X-mode waves also have a transverse component, but near the UH layer, they are mostly longitudinal, making the longitudinal component the component of immediate interest. The simulation produces the time evolution of the longitudinal electric field shown in Fig. 6(a). The temporal evolution of the longitudinal electric field in the homogeneous region, i.e., $|x|$ $\leq 5 \mathrm{~mm}$, can be separated into three distinct phases. Phase A is the

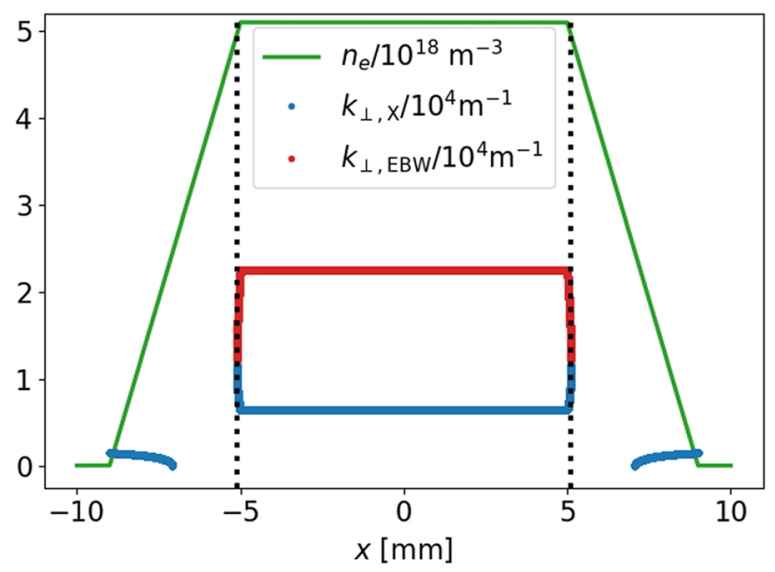

FIG. 5. Density profile for simulations of TPD near the second harmonic UH layer. Red and blue lines are + and - , respectively, of Eq. (3) for $f_{j}=f_{0} / 2=70 \mathrm{GHz}$. The electron temperature is $T=100 \mathrm{eV}$, the magnetic field is $B=B_{y}=2.4 \mathrm{~T}$, and the black dotted lines mark positions of the UH layer of the trapped $f_{0} / 2$ waves. The pair of daughter waves excited in the homogeneous center has a small frequency mismatch and produces two such loops of different sizes.

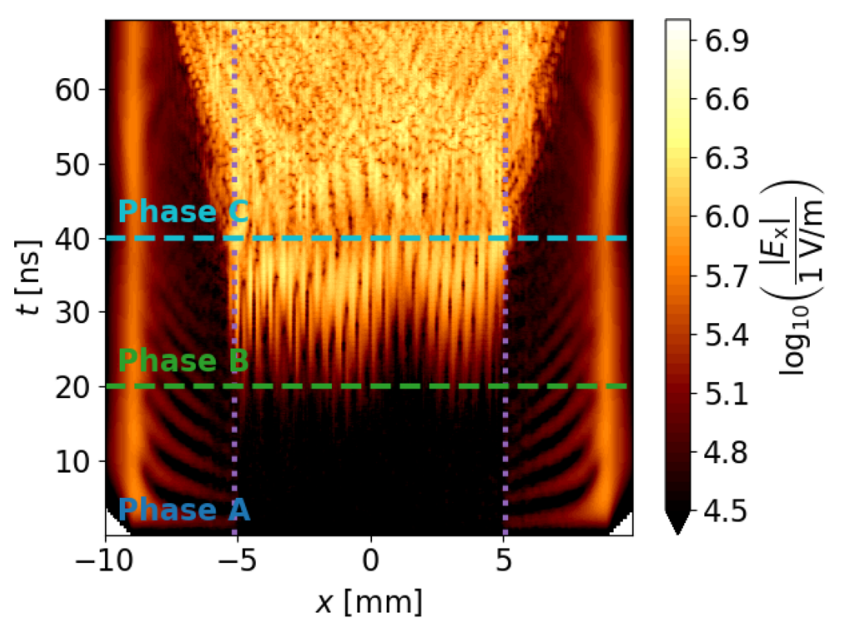

(a)

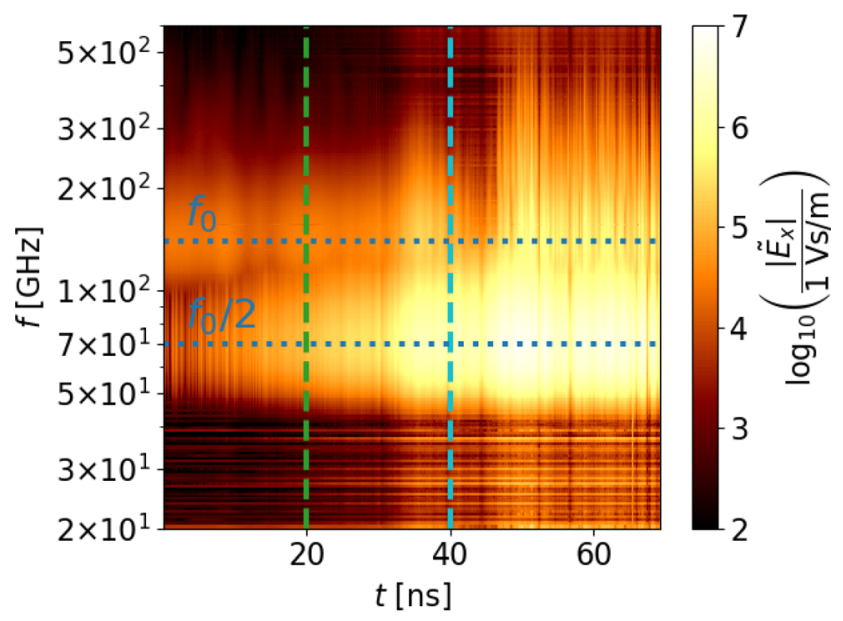

(b)

FIG. 6. The longitudinal electric field component resulting from a simulation of the profile shown in Fig. 5 and a pump intensity of $I_{\text {pump }}=1 \mathrm{~kW} / \mathrm{mm}^{2}$. (a) Longitudinal electric field component in time and space. Colored dashed horizontal lines mark the beginning of the phase as labeled above it, used for reference in the text. Dotted vertical purple lines mark the UH layer for $70 \mathrm{GHz}$ UH waves. The slow oscillations outside the homogeneous region are ion waves (see the text). (b) A CWT of (a) at the $x=0 \mathrm{~mm}$ center line. Dotted horizontal blue lines mark frequencies as labeled above them. Dashed vertical lines indicate phases as shown by corresponding colors in (a).

starting phase where plasma noise dominates. Phase B starts roughly at $t \approx 20 \mathrm{~ns}$ where simple wave patterns appear and grow to a level greater than thermally excited longitudinal waves. Finally, phase $\mathrm{C}$ starts at about $t \approx 40$ ns when the excited waves turn into more complicated patterns. The phases are indicated by the blue, green, and teal labels and lines in Fig. 6(a). It is noted that the excited waves in phase $\mathrm{B}$ seem to stay roughly inside the indicated trapping region of $70 \mathrm{GHz}$ waves, but in phase $\mathrm{C}$, waves start propagating further out. The waves in phase $\mathrm{B}$ are expected to be the trapped primary daughter waves, whereas the escaping waves in phase $C$ could be daughter waves of 
secondary decay processes and will be referred to as secondary daughter waves. The waves will be discussed in greater detail in the next paragraphs. Other noticeable features of the longitudinal field are the slow waves and fields near the boundaries. The frequency is found to be $250 \pm 30 \mathrm{MHz} \approx f_{\mathrm{pi}}$ for $x=7 \mathrm{~mm}$ and $2 \mathrm{~ns}<t<22 \mathrm{~ns}$, and the oscillations are therefore likely to be ion plasma waves from the ions that are able to travel further out than the electrons. These slow waves, however, are independent of the pump wave intensity and are therefore not the focus of this investigation. Inspecting instead the transverse component, little is seen other than the pump wave, and this figure is therefore omitted.

Along the $x=0 \mathrm{~mm}$ centerline, a continuous wavelet transform (CWT) into frequency is applied to the longitudinal electric field and is shown in Fig. 6(b). At the pump frequency, the level is found to be fairly stable until $t \approx 30 \mathrm{~ns}$. At half the frequency, the level is seen to build up several orders of magnitude until the same $t \approx 30 \mathrm{~ns}$ after which a correlated slow oscillatory behavior starts at both pump and half pump frequencies. This supports the expectation that the waves in phase B of Fig. 6(a) are the primary daughter waves, excited by the pump.

Applying fast Fourier transforms (FFTs) to the longitudinal electric field from $x, t$ into $k$, $f$, waves near the $\mathrm{UH}$ frequency throughout the homogeneous region are investigated in phases A, B, and C. In phase A, Fig. 7(a), the thermally excited X and EBW dispersion lines are observed. The dispersion relation in Eq. (3) is also shown in Fig. 7(a). These thermally excited lines are independent of pump intensity.

In phase B, Fig. 7(b), four strong peaks have appeared along the dispersion lines, all with peak values at $70.00 \pm 0.05 \mathrm{GHz}$. The peaks are labeled $\mathbf{a}, \mathbf{a}^{\prime}, \mathbf{b}$, and $\mathbf{b}^{\prime}$. Referring to Figs. 4 and 5, TPD in the homogeneous should produce a pair of EBWs at 69.98 and $70.02 \mathrm{GHz}$, which is inside the error of the observed EBW peaks labeled $\mathbf{a}$ and $\mathbf{a}^{\prime}$. TPD could also produce a pair of X-mode waves at 69.94 and $70.06 \mathrm{GHz}$, which is just outside the error margin for the peaks labeled $\mathbf{b}$ and $\mathbf{b}^{\prime}$ and therefore not in agreement with theory. The X-mode peaks may simply be the EBW daughter waves, which have been linearly converted at the UH layers. However, visually inspecting the spectra, the X-mode peaks seem slightly shifted in frequency compared to the EBWs, which they should not be if they were simply linearly converted. Perhaps the growth rate of the EBWs is greater, and this shifts the observed maxima of the X-mode peaks toward $70 \mathrm{GHz}$. A better resolution might have revealed separate peaks, but the rapid evolution of the electric field sets a limit on the resolution that can be attained in these spectra.

In phase $\mathrm{C}$, Fig. 7(c), the general level along the $\mathrm{X}$ and $\mathrm{EBW}$ branches has increased such that it is at the same level as the primary daughter waves in phase $\mathrm{C}$, which do not appear to have increased further in intensity. This, along with Fig. 6, suggests that the TPD daughter waves have exceeded the threshold of secondary wave interactions, saturating the level of the primary daughter waves. The secondary daughter waves at lower frequencies are able to travel further out of the density bump before it meets the UH layers, and this agrees well with observations in Fig. 6(a). The secondary wave interactions might be PDIs involving ion modes, which ideally produce distinct peaks all along the UH branches. However, due to the low frequency resolution attainable with such rapid evolution of the spectrum, individual peaks are not observed, but rather a general lift of the level throughout the dispersion lines can be seen in this figure. We note that the EBW

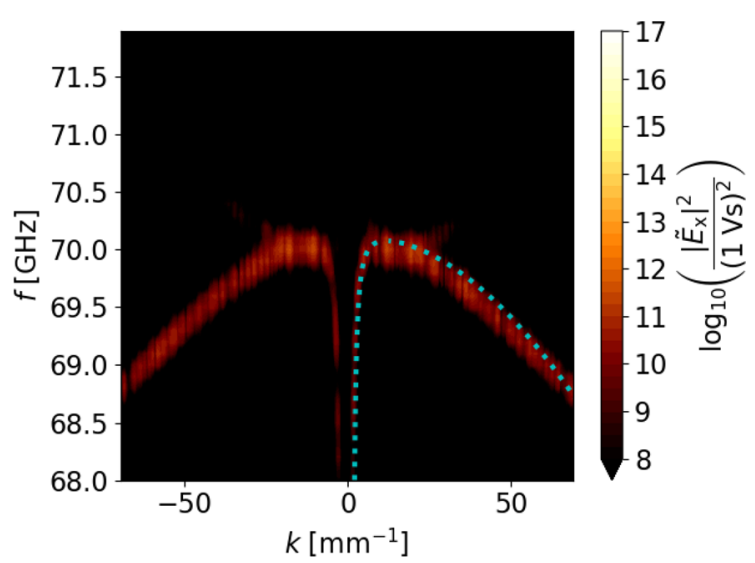

(a)

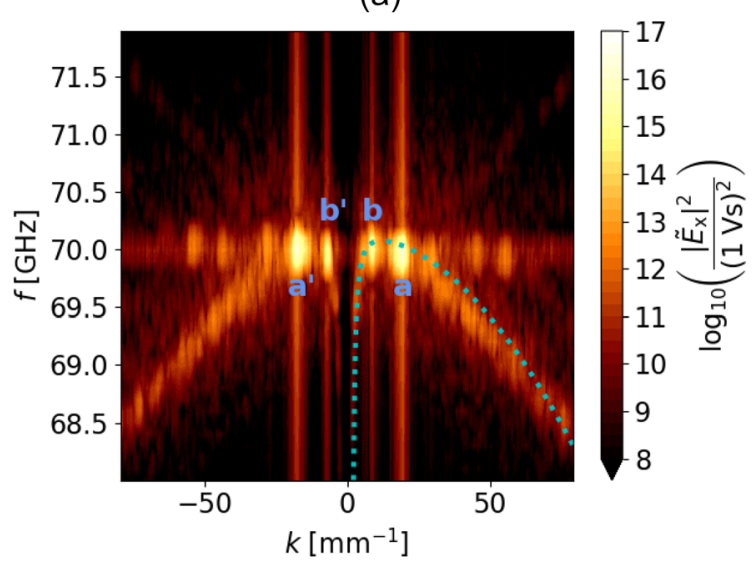

(b)

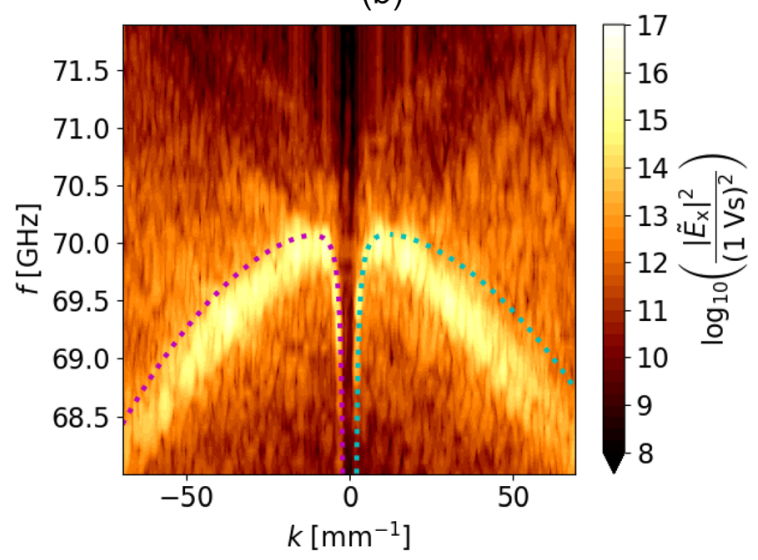

(c)

FIG. 7. FFTs into $k$ - and $f$-space of the longitudinal electric field in the homogeneous region shown in Fig. 6(a) during different phases as indicated in the subcaptions. Cyan dotted lines show positive solutions to Eq. (3). Bold blue letters are labels for nearby peaks mentioned in the text. (a) FFT for $-5 \mathrm{~mm}<x<5 \mathrm{~mm}$ and the time interval $0 \mathrm{~ns}<t<10 \mathrm{~ns}$, corresponding to phase A in Fig. 6(a). (b) FFT for $-5 \mathrm{~mm}$ $<x<5 \mathrm{~mm}$ and the time interval $25 \mathrm{~ns}<t<35 \mathrm{~ns}$, corresponding to phase $\mathrm{B}$ in Fig. 6 (a). (c) FFT for $-5 \mathrm{~mm}<x<5 \mathrm{~mm}$ and the time interval $50 \mathrm{~ns}<t<60 \mathrm{~ns}$, corresponding to phase $\mathrm{C}$ in Fig. 6(a). The added dotted magenta line is the dispersion relation for the elevated mean electron temperature of $120 \mathrm{eV}$ in this interval. 
branches no longer follow the dispersion relation based on initial parameters, possibly because the excited waves modify the electron distribution function. Averaging the electron temperature in the homogeneous region as provided by $\mathrm{EPOCH}$ does indicate an increase, which produces a slightly better fitting dispersion line. Electron heating due to high amplitude UH waves has previously been reported in simulations. ${ }^{33}$ Comparing FFTs of the transverse electric field in the outer homogeneous side regions, i.e., for $|x| \geq 9 \mathrm{~mm}$ in Fig. 5, the transmitted pump intensity drops by about $10 \%$ during the primary instability, suggesting that a significant amount of energy might be absorbed in such a non-monotonic density profile.

To support the idea that the waves discussed above are excited through a TPD instability, a simulation at half density is run. The density profile is shown in Fig. $8(\mathrm{a})$, but $f_{0} / 2=70$ for $\mathrm{GHz} \mathrm{X}$-mode, and EBWs now do not propagate in the homogeneous region. This is because the UH frequency for this profile is $68.6 \mathrm{GHz}$ in the homogeneous region, and it is therefore not possible for the pump to decay through TPD into two trapped UH waves. Looking at Fig. 4, decay into one trapped $\mathrm{UH}$ wave and one escaping electromagnetic wave is possible. This type of parametric decay is also known as stimulated Raman scattering ${ }^{40}$ and is expected to have a lower growth rate since one of the daughter waves is convected away from the decay region, similar to Ref. 51. At the pump intensity used for the previous simulations, there were indications of a trapped daughter wave, but its growth rate was rather small and would require a very long simulation to be run. Therefore, the simulation was run again with the pump intensity increased by a factor of ten to $I_{\text {pump }}=10 \mathrm{~kW} / \mathrm{mm}^{2}$, and an FFT spectrum at a relatively late $55 \mathrm{~ns}<t<65 \mathrm{~ns}$ is shown in Fig. 8(b). As the density has been decreased, the UH layer is now found at a lower frequency, and the dispersion lines are found lower in the plot compared to the previous simulation. The spectrum shows a peak on the positive slow X-mode branch at $68.45 \pm 0.05 \mathrm{GHz}$, labeled $\mathbf{c}$, and one fast X-mode peak at $71.55 \pm 0.05 \mathrm{GHz}$, labeled $\mathbf{c}^{\prime}$. From Fig. 4, it is found that decay in the homogeneous center region produces the same types of waves at frequencies 68.47 and $71.53 \mathrm{GHz}$, which agrees well with observations. We note that the scale in Fig. 8(b) is different from Fig. 7(c) in spite of a greater pump intensity, confirming that this decay has a much lower growth rate. However, $70 \mathrm{~ns}$ is a very short window of time assuming that the non-monotonic density profile is caused by MHD modes.

Increasing the density, on the other hand, should still allow for TPD to take place as indicated in Fig. 9(a) where the density has been doubled. The resulting spectrum in Fig. 9(b) now shows a situation where the UH layer is at higher frequency. Once again, there are two $\mathrm{X}$-mode and two EBW peaks near $70 \mathrm{GHz}$. This time, Eqs. (1)-(3) predict a pair of EBWs at 69.88 and $70.12 \mathrm{GHz}$ and that the low frequency wave has positive $k$, which agrees with the observed values $69.90 \pm 0.05 \mathrm{GHz}$, d, and $70.10 \pm 0.05 \mathrm{GHz}, \mathbf{d}^{\prime}$, with the lower frequency wave corresponding to positive $k$. However, the pump may also decay into two X-mode waves at 67.44 and $72.56 \mathrm{GHz}$, the high frequency one having positive $k$, which are not observed, possibly due to a lower growth rate. The X-mode peaks that are observed, $\mathbf{e}$ and $\mathbf{e}^{\prime}$, lie at the same frequencies as the observed EBW peaks, suggesting that these are simply linearly converted from the EBWs.

\section{EFFECT OF IONS ON FURTHER DECAY PROCESSES}

The TPD instability involves only electron waves and therefore should not depend on ion motion. The secondary PDIs, which were

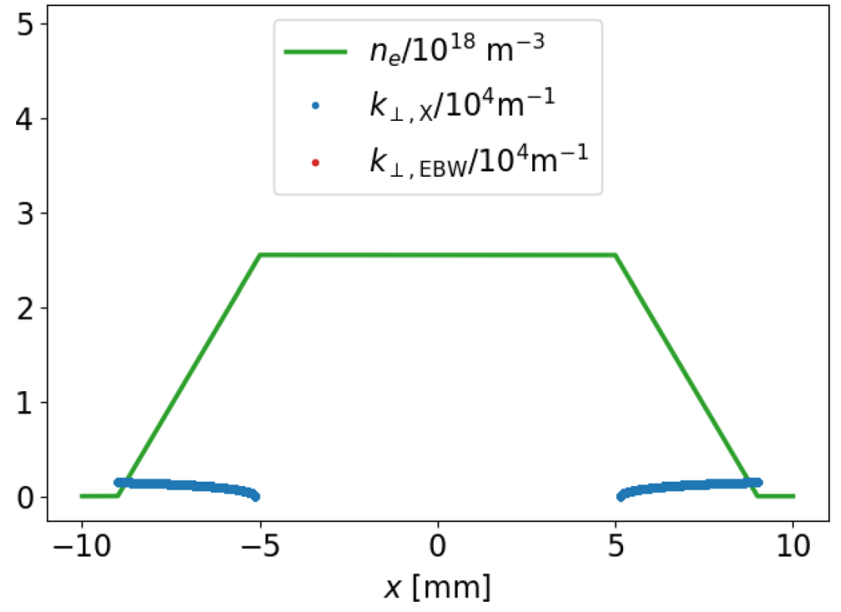

(a)

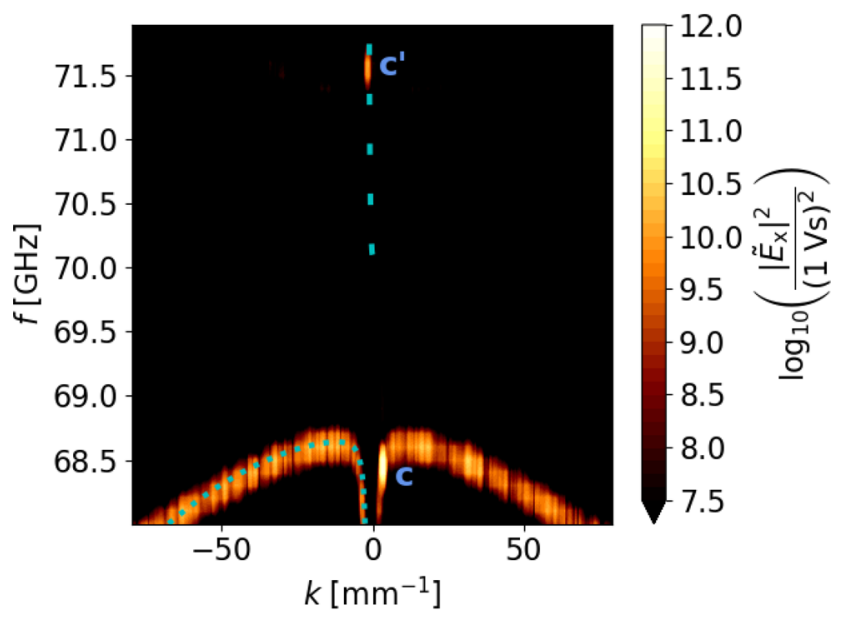

(b)

FIG. 8. Density profile and FFT spectrum of the longitudinal electric field for a simulation where TPD is not possible (compare with Fig. 5). (a) A density profile where half frequency UH waves cannot propagate in side, and TPD is therefore not possible. Waves at lower frequencies may still be trapped inside the density bump. (b) $k$ - and $f$-space for $55 \mathrm{~ns}<t<65 \mathrm{~ns}$ with the profile shown in Fig. 8(a) and an intensity of $I_{\text {pump }}=10 \mathrm{~kW} / \mathrm{mm}^{2}$; one trapped, $\boldsymbol{c}$, and one escaping wave, $\mathbf{c}^{\prime}$, are excited. The dotted cyan line in the lower half shows dispersion lines for trapped waves in negative $k$, Eq. (3), while the more spaced out dotted line in the top half indicates escaping $\mathrm{X}$-mode waves in negative $k$, Eq. (2). This spectrum is not directly comparable to Fig. 7(c) due to a change in both density and pump intensity.

seen to populate the lower frequency parts of the UH spectrum shown in Fig. 7(c), are expected to be an interaction between UH waves and ion waves, such as LH or IBWs, which gradually downshift the trapped waves. Without ion dynamics, the secondary PDIs therefore should not take place. To confirm this, the simulation presented in Figs. $5-7$ is rerun but with the ions taken out of the particle pusher algorithm, making them immobile. As a result, ion waves will not be able to propagate and parametric instabilities involving ion wave dynamics will not be observed. Looking at the temporal and spatial 


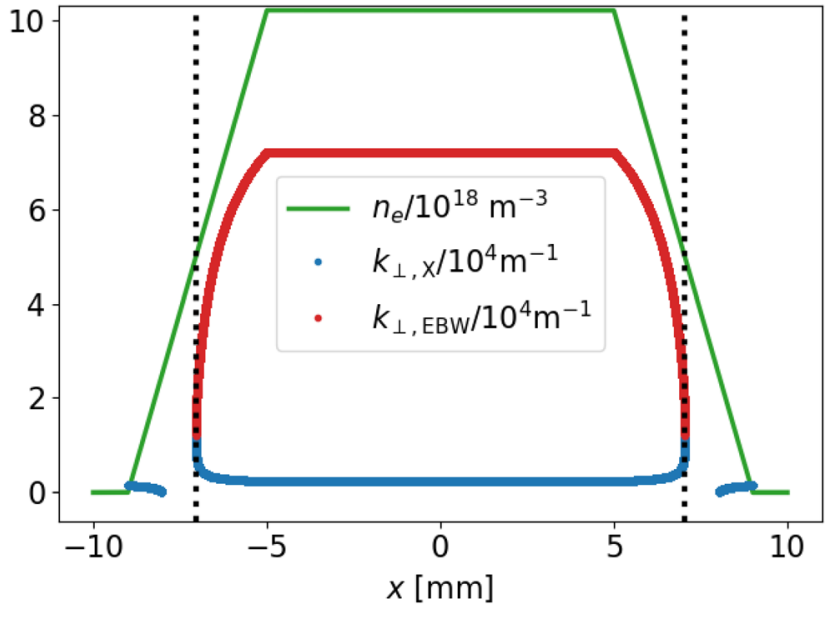

(a)

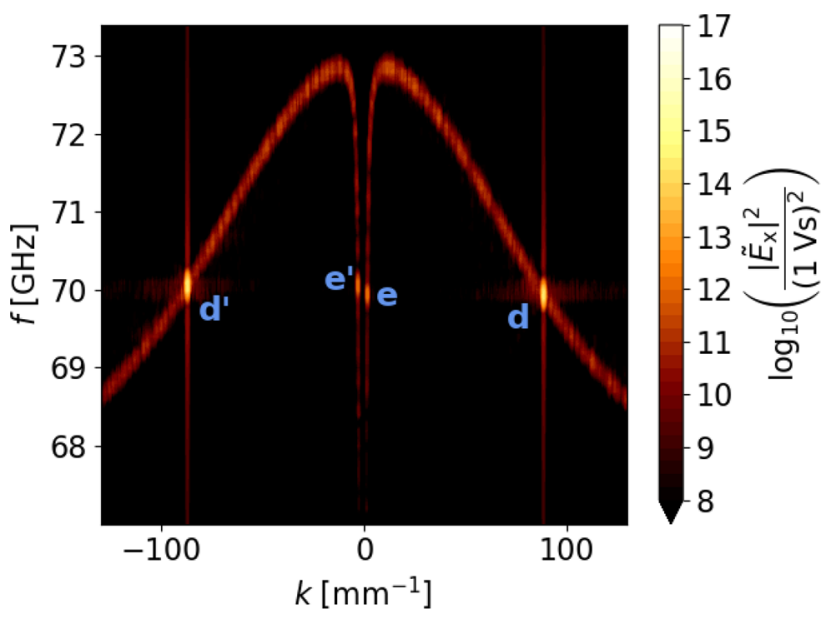

(b)

FIG. 9. Density profile and FFT spectrum of the longitudinal electric field for a simulation with a higher density setup further from the second harmonic UH layer (compare with Fig. 5). (a) Density profile with the red and blue lines being + and - , respectively, of Eq. (3) for $f_{j}=f_{0} / 2=70 \mathrm{GHz}$. The $\mathrm{UH}$ layers are indicated by the vertical dotted lines. (b) $k$ - and $f$-space for $I_{\text {pump }}=1 \mathrm{~kW} / \mathrm{mm}^{2}$ and $25 \mathrm{~ns}<t<35$ ns like in Fig. 7 (b). Bold blue letters are labels for nearby peaks mentioned in the text.

evolution of the longitudinal electric field in Fig. 10(a), the slow oscillations near the boundaries that are seen in Fig. 6(a) have now disappeared, confirming that they were ion waves. The TPD daughter waves are still excited as they should since they are three wave interactions between electron waves. The third phase, where other wave interactions come into play and the waves start spreading out, is not seen in this simulation, confirming that it is PDIs involving both electron and ion waves that cause the cascading of energy to lower frequency. Comparing Fig. 7(c) with Fig. 10(b), it is seen that without the ion dynamics, the waves excited from TPD still clearly stand out, and their peaks have continued to grow as the saturation mechanism provided by interactions with ion waves is no longer possible.

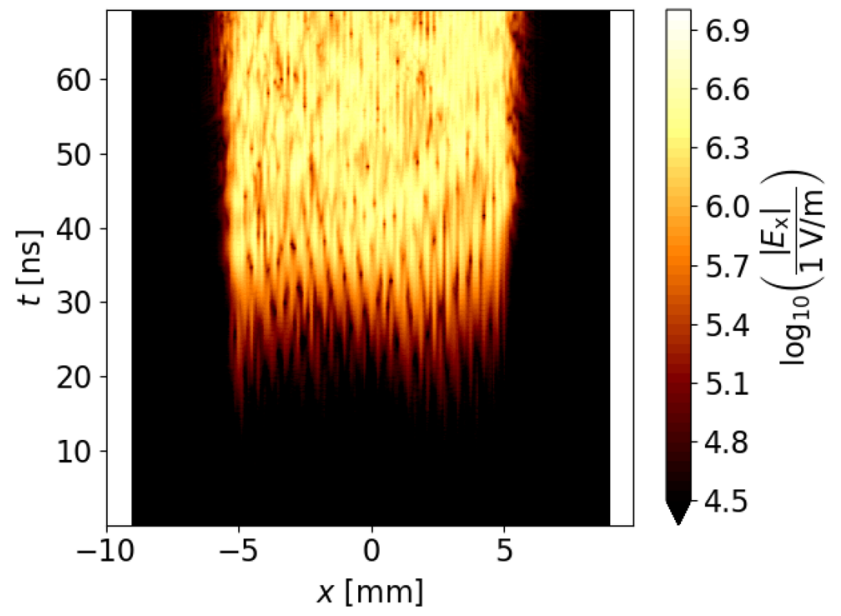

(a)

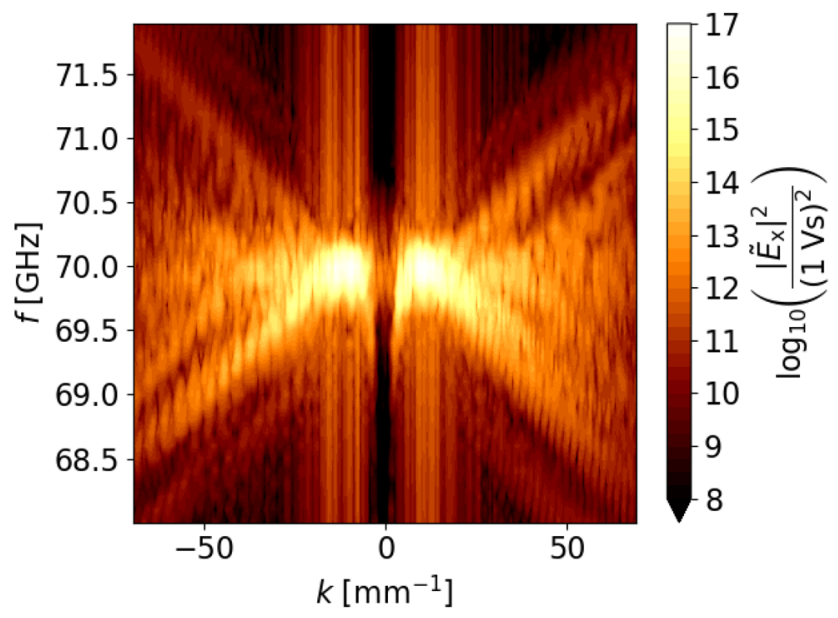

(b)

FIG. 10. Resulting longitudinal electric field from a simulation with immobile ions. Parameters are otherwise identical to those in Fig. 5. (a) Evolution of the longitudinal electric field for immobile ions in time and space [compare with Fig. 6(a)] where plasma parameters are the same but ions are mobile. (b) $k$ - and $f$-space for $50 \mathrm{~ns}$ $<t<60$ ns [compare with Fig. 7(c)] where plasma parameters are the same but ions are mobile.

\section{DENSITY PROFILE HAS LITTLE EFFECT ON GROWTH}

Until this point, it has been assumed that TPD was taking place in the homogeneous region because this is where the pump intensity threshold is the lowest. In the following, we compare the hard density profile from before to a number of inhomogeneous profiles seen in Fig. 11 to investigate the effect of the density profile on the decay rate. Furthermore, the size of the trapping region is varied by changing the profile to see if that has any influence on the temporal evolution of the trapped waves. All the inhomogeneous profiles are on the form $n(x)=\exp \left(-\left|x / \ell_{\mathrm{n}}\right|^{\gamma}\right)$, where $\ell_{\mathrm{n}}$ and $\gamma$ are listed in Table I.

The super-Gaussian profile is very similar to the hard profile, being essentially homogeneous in the center. Comparing Fig. 12(a), which is a cropped duplicate of Fig. 6(a) and Fig. 12(b), the dynamics 


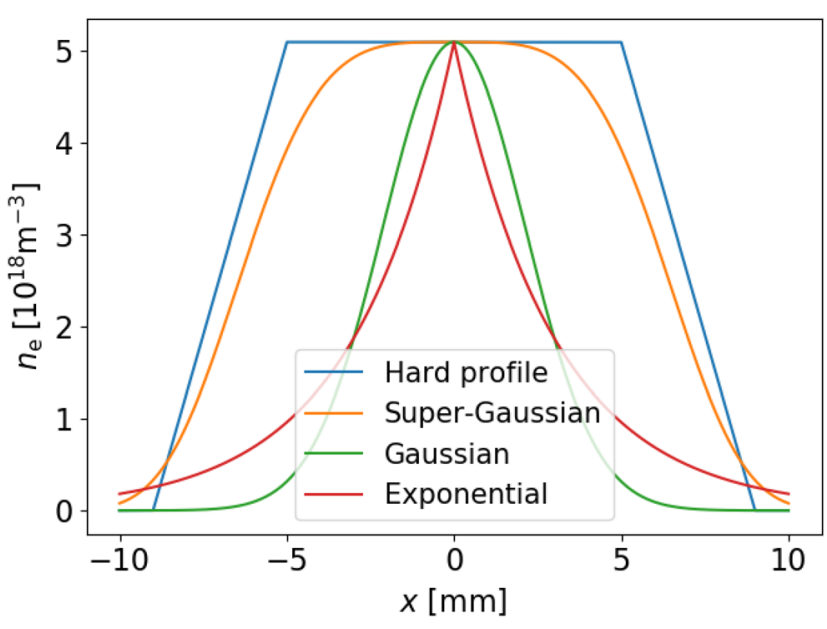

FIG. 11. Different density profiles to be compared. The hard profile refers to the simulation based on Fig. 5. The remaining profiles are of the form $n(x)=\exp \left(-\left|x / \ell_{n}\right|^{\gamma}\right)$ with the relevant parameters shown in Table I. Resulting longitudinal fields using an intensity of $I_{\text {pump }}=1 \mathrm{~kW} / \mathrm{mm}^{2}$ are shown in Fig. 12.

are also very similar albeit the TPD waves are confined to a narrower region as the $\mathrm{UH}$ layers of $\sim 70 \mathrm{GHz}$ waves are closer than in the hard profile. For the Gaussian profile in Fig. 12(c), the center can no longer be considered homogeneous over a $70 \mathrm{GHz}$ X-mode wave, but the TPD daughter waves still build up to the same level, on the same timescale and with the same three phases placed roughly at the same times. As the bounce time of the TPD daughter waves is greatly reduced by going from the hard profile to the Gaussian, this suggests that the growth rate of TPD daughter waves inside the density bump is largely independent of the homogeneous region and of the size of the density bump. The TPD theory that is believed to explain the strong scattering during second harmonic ERCH is based on excitation of eigenmodes of the profile, which satisfies conditions on the wave vector similar to Bohr-Sommerfeld quantization. ${ }^{50}$ A trapped wave should obtain an integer multiple of $2 \pi$ phase change as it performs a round trip with two conversions in the trapping region to interfere constructively with itself. By choosing a profile such as Fig. 5, we have made decay into the homogeneous region more favorable, i.e., primary daughter waves that might not be eigenmodes. However, the time evolution of the longitudinal field in the Gaussian profile, where one would expect decay into eigenmodes to dominate, does not show any sign of a reduced growth rate. Finally, for the exponential profile in Fig. 12(d), the peak density does exceed that of the UH layer for $70 \mathrm{GHz}$ waves, but it would appear that the $\mathrm{UH}$ layers are so close that the daughter waves are not supported inside the density bump and no buildup of waves is observed.

TABLE I. Parameters for the density profiles in Fig. 11, which are on the form $n(x) \propto \exp \left(-\left|x / \ell_{n}\right|^{\gamma}\right)$. Resulting longitudinal fields using an intensity of $l_{\text {pump }}=1 \mathrm{~kW} / \mathrm{mm}^{2}$ are shown in Fig. 12 .

\begin{tabular}{lcc}
\hline \hline Profile & $\gamma$ & $\ell_{\mathrm{n}}(\mathrm{mm})$ \\
\hline Super-Gaussian & 4 & 7 \\
Gaussian & 2 & 3 \\
Exponential & 1 & 3 \\
\hline \hline
\end{tabular}

\section{PUMP INTENSITY COMPARISON}

By scanning the pump intensity of the density profile shown in Fig. 5, the growth rate of the first TPD daughter waves is investigated. Averaging the square longitudinal electric field over the homogeneous region, the time evolution of the resulting signal is assumed to first be dominated by a background level due to plasma fluctuations followed by a phase where an absolute instability gives rise to an exponential growth. Eventually, other processes become important, but only the growth rate of the initial TPD instability is sought, so a function of the form $\phi(t)=\left\langle E_{0}^{2}\right\rangle+a \exp \left(2 \gamma_{\mathrm{TPD}} t\right)$ is fitted to the mean squared longitudinal electric field up until it reaches a level of $10^{10}(\mathrm{~V} / \mathrm{m})^{2}$. Figure 13(a) shows a number of signals for different pump intensities with $\phi$ fits. As a general feature, the squared mean fields start at a constant level, which seems to vary with pump intensity. An exponential growth takes over next, growing several orders of magnitude. Finally, the field overshoots a threshold level and saturates at a lower level, arguably because threshold levels of secondary PDIs saturate the growth inside the homogeneous region. The overshoots also depend on pump intensity, but they appear to relax, following the same asymptotic curve. Plotting the fitted growth rates against pump intensities in Fig. 13(b), they are found to go as $2 \gamma_{\mathrm{TPD}} \propto I_{\text {pump }}^{0.5}$. This agrees with Ref. 50 if the pump is assumed to be of infinite width but finite intensity. A similar approach applied to the low density simulation presented in Fig. 8, where Raman scattering was observed instead of TPD, gives a growth rate of $\gamma_{\mathrm{TPD}}=5.0$ $\times 10^{-2} \mathrm{~ns}^{-1}=5.7 \times 10^{-5}\left(2 \pi f_{0}\right)$ at an increased pump intensity of $I_{\text {pump }}=10 \mathrm{~kW} / \mathrm{mm}^{2}$.

\section{ESCAPING WAVES}

Once the primary daughter waves have been excited, they can interact with other waves in the plasma. One of the simplest wave interactions to observe is recombination with the pump wave. The resulting wave would be an escaping X-mode wave at approximately $3 / 2 f_{0}=210 \mathrm{GHz}$. The possibility of observing the $3 f_{0} / 2$ signal due to trapping of TPD daughter waves in a magnetically confined plasma has also been proposed analytically. ${ }^{56}$ On one hand, the escaping $3 f_{0} / 2$ waves provide a diagnostic opportunity as indications of trapped waves may be observed without having to look near the pump frequency where stray radiation from the gyrotrons can damage sensors. While backscattered waves in the pump frequency range is the result of tertiary PIs, the $3 f_{0} / 2$ waves are the result of just 2 PIs, both involving the known pump wave, and may therefore provide a more direct indication of trapped UH waves. On the other hand, the escaping waves pose a risk for detectors that happen to be sensitive to the particular frequency range but might not be taking radiation due to PIs into account.

The difference in $k$ for such a process, $\Delta k=k_{0}+k_{1}-k_{210}$, can be seen in Fig. 14(a) for left and right propagating escaping waves as a function of TPD daughter frequency. A match, i.e., $\Delta k=0$, is only possible for a left propagating wave, escaping back where the pump came from. Figure 14(b) shows a comparison of the $f$-spectrum near $210 \mathrm{GHz}$ at the boundaries of the domain. Both spectra display similar trends except in the immediate vicinity of $210 \mathrm{GHz}$ where a large peak is present in the spectrum at the left boundary, agreeing with the $k$ match analysis. 


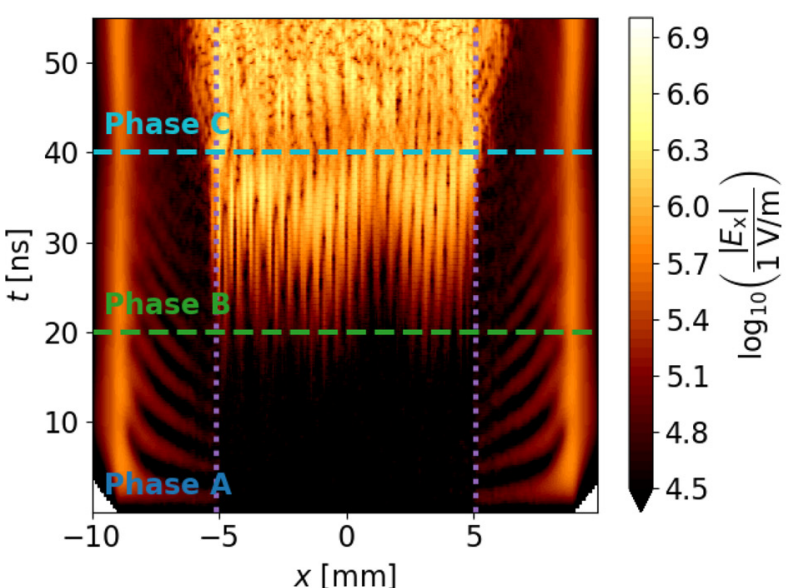

(a)

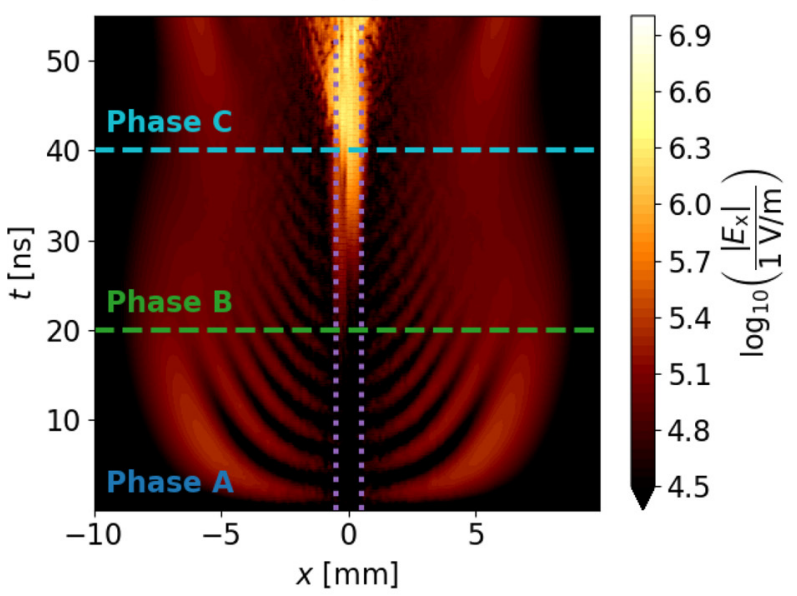

(c)

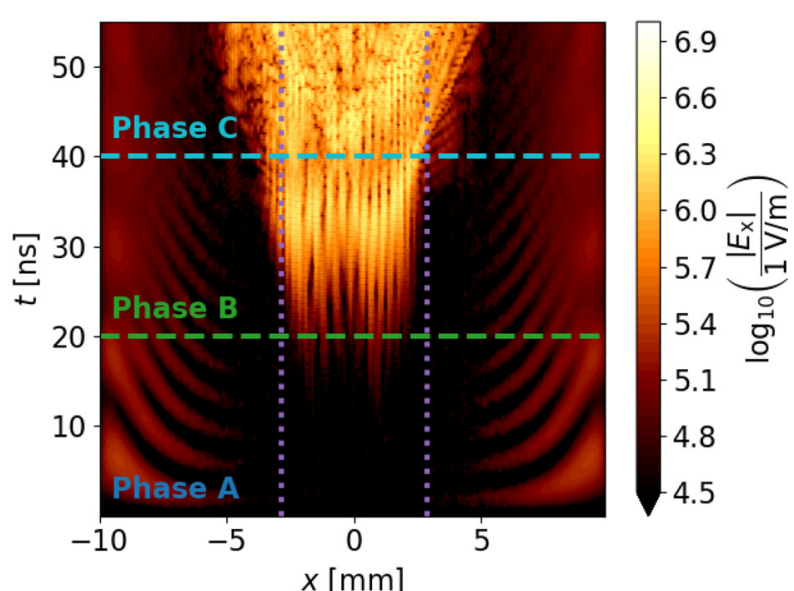

(b)

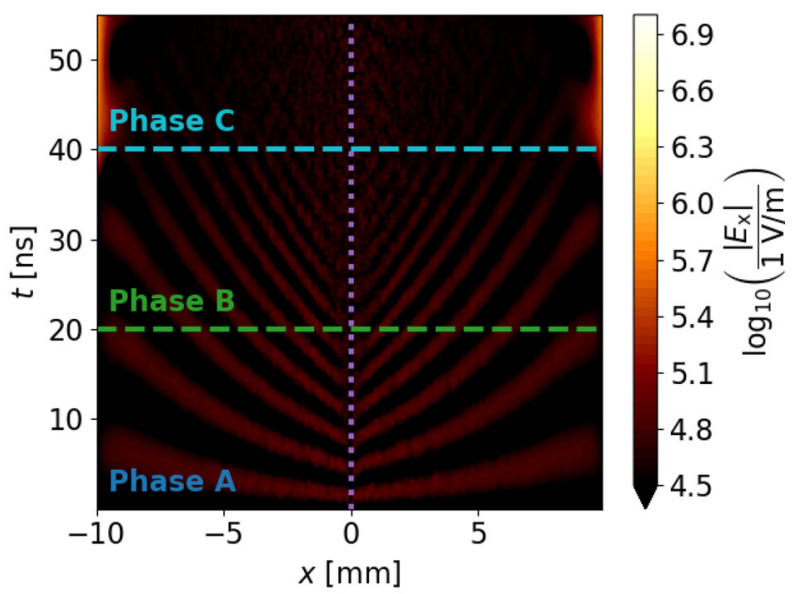

(d)

FIG. 12. Evolution of the longitudinal electric field in time and space for four density profiles shown in Fig. 11. The marked phases refer to the phases observed in Fig. 6(a), while the purple lines conform to the individual plots, showing the confines of the trapping region for $70 \mathrm{GHz}$ waves. (a) The hard profile; a cropped duplicate of Fig. 6(a). (b) The super-Gaussian profile with $\gamma=4$ and $\ell_{n}=7 \mathrm{~mm}$. (c) The Gaussian profile with $\gamma=2$ and $\ell_{n}=3 \mathrm{~mm}$. (d) The exponential profile with $\gamma=1$ and $\ell_{n}=1 \mathrm{~mm}$.

\section{2D SIMULATIONS}

The presented simulations have until now been 1D. Besides the concern that homogeneous regions would lead to excitation of primary daughter waves that were not eigenmodes, PDIs were restricted to the $k$-match condition $\left|k_{0}\right|=\left|k_{1} \pm k_{2}\right|$. Through simple geometric arguments, it can be shown that if both the inequalities $\left|\mathbf{k}_{0}\right|$ $\leq\left|\mathbf{k}_{1}\right|+\left|\mathbf{k}_{2}\right|$ and $\left|\mathbf{k}_{0}\right| \geq|| \mathbf{k}_{1}|-| \mathbf{k}_{2}||$ are satisfied, it will be possible to find a set of angles in $2 \mathrm{D}$ such that $k$-match is possible. This means that TPD can lead to wave propagation at a continuum of angles, perhaps allowing for other decay into eigenmodes to take place in the presence of a homogeneous region. A $2 \mathrm{D}$ simulation is devised to investigate if new wave dynamics arise with an additional dimension to propagate in. For this simulation, an initially entirely homogeneous $y$-direction has been added. The domain width along the $y$-direction is $w_{\mathrm{y}}=1.0375 \mathrm{~mm}$ with $n_{\mathrm{y}}=83$ grid points and periodic boundary conditions. The domain width along the $y$-direction is chosen such that the slightly shorter $69.98 \mathrm{GHz}$ daughter wave from the simulation outlined in Fig. 5 can fit four wavelengths if it was to propagate parallel to the $y$-direction. This is to avoid strong destructive interference of trapped waves with a nonzero $y$-component of the wave vector. The number of grid points is chosen such that the grid spacing in the $y$ direction is equal to that of the $x$-direction. The direction of the magnetic field is changed to point in the $z$-direction, i.e., $B=B_{\mathrm{z}}$, so that both the $x$ - and $y$-directions are perpendicular to the magnetic field. The pump wave has to be transverse, so its electric field is now polarized in the $y$-direction. Parameters along the $x$-direction are kept mostly unchanged with the exception of the number superparticles now given by $n_{\text {part }}=3 \times 10^{3} n_{\mathrm{x}} n_{\mathrm{y}}$. For an intensity of $I_{\text {pump }}=1 \mathrm{~kW} / \mathrm{mm}^{2}$, the growth rate is estimated in the same manner as was discussed in Sec, VI, averaging now not only over $-5 \mathrm{~mm}$ $<x<5 \mathrm{~mm}$ but also along the entire $y$-axis. Unlike 1D, which used all available data points, the $2 \mathrm{D}$ fit is applied to a signal sampled with a time step of $0.05 \mathrm{~ns}$ due to the increase in data size, but this time step should be sufficient for the targeted timescale. As is seen in Fig. 15(a), 


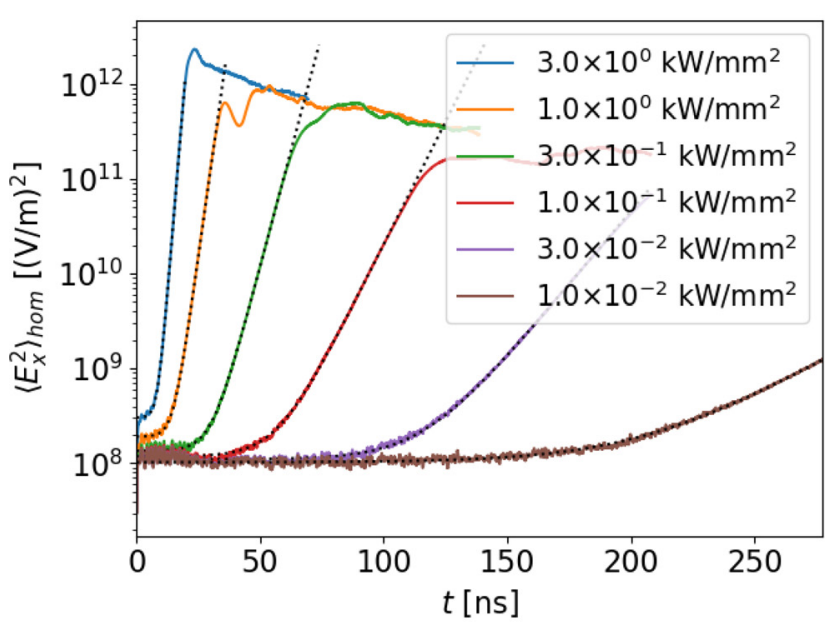

(a)

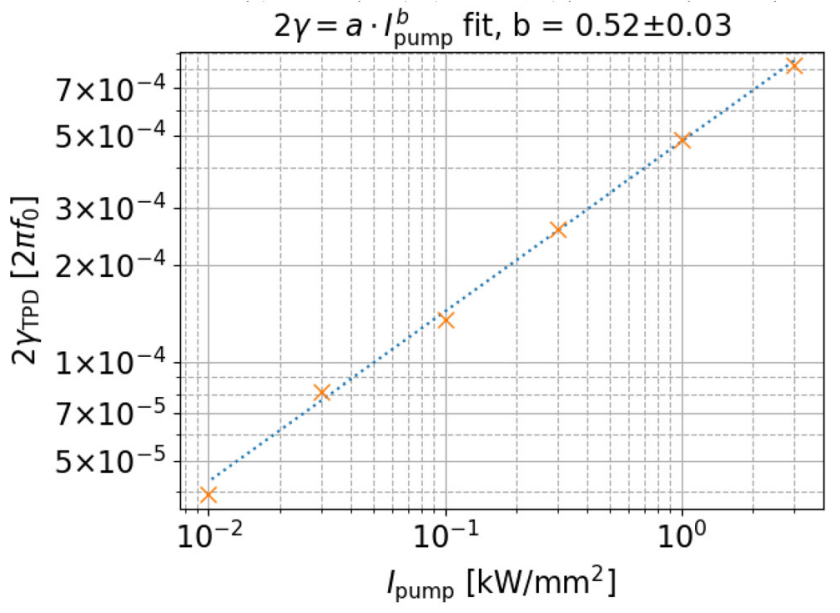

(b)

FIG. 13. A scan of pump intensity for the profile shown in Fig. 5. Then, for each simulation, the growth rate of the primary decay instability is determined, and a power law is fitted to the found growth rate plotted against the pump intensity. (a) Mean squared longitudinal electric field averaged over the homogeneous center of the domain for a number of pump intensities. The plotted curves are averaged over 50 time steps. The dotted black lines are fits of the form $\phi(t)=\left\langle E_{0}^{2}\right\rangle+a \exp \left(2 \gamma_{T P D} t\right)$. (b) A plot of the fitted growth rates in Fig. 13(a) against pump intensity shown with orange crosses. The dotted blue line is the fit indicated above the plot.

allowing waves to propagate at an angle to the pump does not change the growth rate significantly. Figure 15(b), however, shows that waves with wave vector components in the $y$-direction are also excited. These waves might not contribute much to the estimated growth rate if they are longitudinal. It is, nevertheless, more challenging to include them in the growth rate as the presence of the pump wave elevates the background level significantly, making the growth of an instability less apparent. As suggested by Fig. 15(a), a more stochastic saturated phase similar to phase C in Figs. 6(a) and 7(c) follows at greater $t$, suggesting that $1 \mathrm{D}$ simulations capture the primary and secondary PIs of this $2 \mathrm{D}$ simulation to a fair degree.

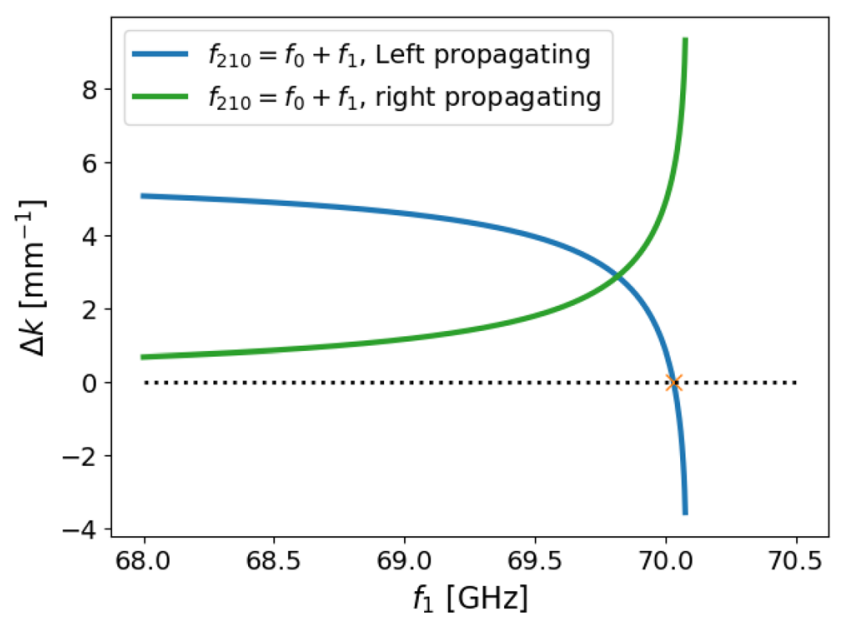

(a)

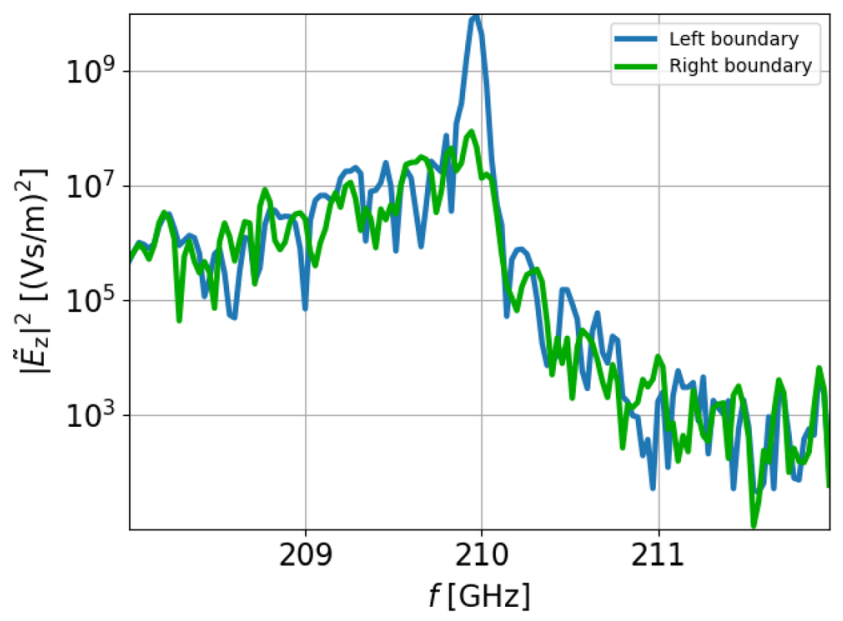

(b)

FIG. 14. Justification for and observation of $3 / 2 f_{0}$ frequency backscattering for the profile shown in Fig. 5 and the simulation shown in Fig. 6(a). (a) A plot of the necessary $k$-match condition for left and right propagating $\sim 210 \mathrm{GHz}$ waves in the homogeneous region of the profile shown in Fig. 5. The $y$-axis shows $\Delta k=k_{0}+k_{1}$ $-k_{210}$, and $\Delta k=0$ means that the selection rules are satisfied; this is marked by the horizontal dotted black line, and an orange cross shows the intersection with the left propagating blue line. (b) The $f$-spectrum at the boundaries of Fig. 5 for $25 \mathrm{~ns}<t<60$ ns near the $3 / 2 f_{0}$ frequency. A much higher peak is present on the left boundary, corresponding to back scattering.

We note that the 2D simulation presented here was purposely made very similar to the $1 \mathrm{D}$ simulations in order to better compare what difference it makes to allow for wave propagation in other directions. Future 2D simulations should explore the effect of a dimension parallel to the magnetic field. This should not affect the primary instability much as the implicated waves are transverse and longitudinal; however, secondary daughter waves may benefit from being able to propagate at an oblique angle to the magnetic field. Similarly, pump waves incident at an angle, and finite beam and bump size should be explored. A finite pump beam size might affect growth rates and thresholds of the TPD instability. ${ }^{4-50}$ 


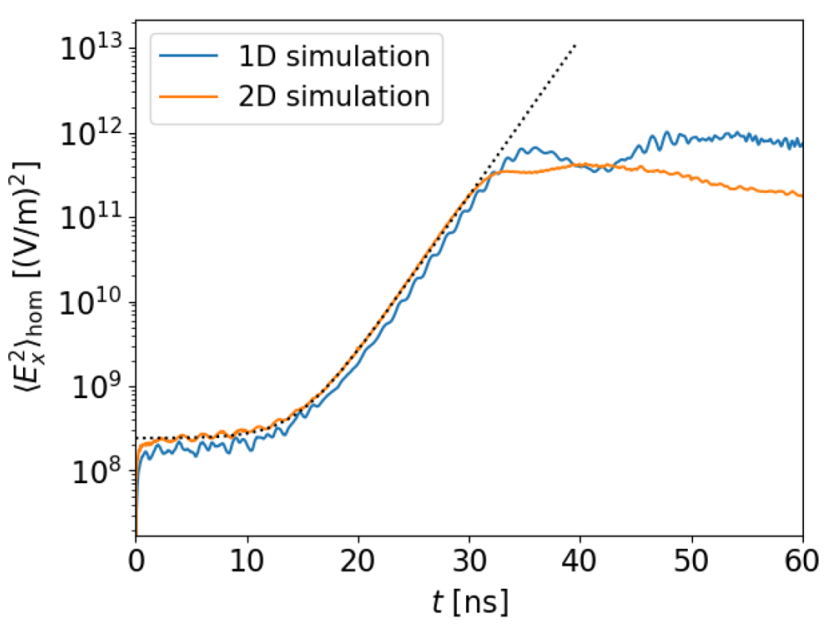

(a)

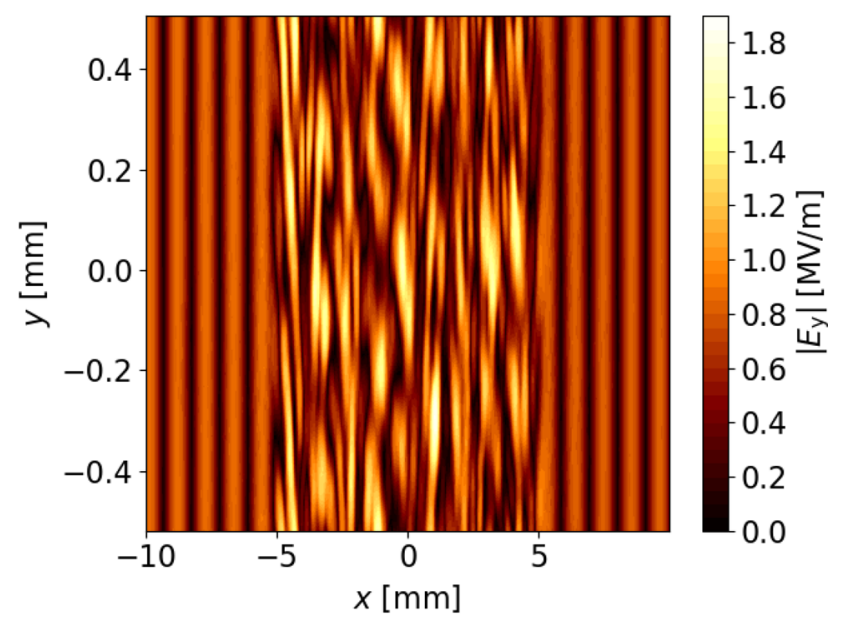

(b)

FIG. 15. 2D simulations using parameters similar to Fig. 5 but with a narrow periodic $y$-direction and the magnetic field pointing in the $z$-direction. (a) Mean squared $x$-component of the electric field averaged over the homogeneous center of the $2 \mathrm{D}$ domain for a simulation with a pump intensity of $I_{\text {pump }}=1 \mathrm{~kW} / \mathrm{mm}^{2}$. The mean squared field is compared to a $1 \mathrm{D}$ simulation of similar parameters, and the growth of the 2D simulations is fitted with an exponential function. The growth rate is found to be $2 \gamma_{\text {TPD }}=4.3 \times 10^{-1} \mathrm{~ns}^{-1}=4.9 \times 10^{-4}\left(2 \pi f_{0}\right)$ for the $2 \mathrm{D}$ simulation. (b) A snapshot of the $y$-component of the electric field at $t=30 \mathrm{~ns}$, after the primary daughter waves have visibly formed. Note that the scales on the $x$ - and $y$-axes are not the same. Unlike the pump wave, the trapped waves not only propagate in the $x$-direction. Compare to the 1D figure in Fig. 6(a).

\section{CONCLUSION}

Using the PIC code EPOCH, we have demonstrated that an Xmode pump wave can excite half frequency trapped daughter waves through TPD, provided that the UH frequency exceeds the daughter wave frequencies. Even when the UH frequency is just below half the pump frequency, stimulated Raman scattering may excite trapped daughter waves, albeit at a much lower growth rate. Trapping implies that the daughter waves can build up and become unstable at low pump intensities as they are not convected out of the decay region. In particular, second harmonic X-mode ECRH is at risk of exciting trapped TPD daughter waves if the gyrotron beam passes a nonmonotonic region, which may be due to islands, filaments, or other mechanisms.

For the first time, we have numerically validated the recent hypothesis that non-monotonic fusion plasmas may facilitate PDIs at reduced gyrotron power thresholds using fully kinetic simulations. A comparison of shapes and sizes of density profiles showed unexpectedly that absorption of gyrotron power into trapped waves can occur even in structures smaller than $1 \mathrm{~cm}$ with little effect on the growth rate of the primary daughter waves. Furthermore, the addition of a homogeneous region also had little effect on the growth rate. We found that the transmission of the pump intensity dropped by about $10 \%$ during the primary instability. For a $1 \mathrm{MW}$ gyrotron, this could potentially account for a significant waste of energy, which is deposited in the wrong parts of the plasma. We characterized the growth rate of the primary instability vs pump intensity in $1 \mathrm{D}$ and found an approximate square-root dependence. There was no sign of a low pump intensity threshold, but this is likely due to the lack of loss mechanisms in 1D such as diffraction of the trapped waves along the magnetic field lines.

Extending to $2 \mathrm{D}$ with another dimension perpendicular to the magnetic field did not affect the growth rate significantly. For gyrotron power levels, the simulations show a fast initial TPD growth timescale on the order of $10 \mathrm{~ns}$. The primary instability saturates on the order of 100 ns due to secondary PDIs. The secondary PDIs depend on ion dynamics, coupling to IBWs to populate $\mathrm{UH}$ waves at various frequencies.

We have shown that the primary daughter waves are able to recombine with the pump into waves at $\sim 3 f_{0} / 2$, which are not trapped but instead travel back where the pump wave came from. This may pose a problem for microwave sensitive equipment not shielded against such waves but could provide an opportunity to diagnose the trapped waves without having to measure in the frequency range of the pump, where stray radiation from multiple gyrotrons makes the task challenging. The emitted $3 f_{0} / 2$ waves are also easier to interpret compared to the signals near the pump frequency since they are the result of direct scattering of the trapped waves.

In conclusion, we have demonstrated that TPD of ECRH beams can occur in small scale density perturbation of magnetically confined fusion plasmas at power levels typically applied in present day experiments. This can potentially modify power deposition profiles and hamper microwave diagnostics. In the light of the present article, PIC codes provide a tool to assess such concerns in fusion experiments.

\section{ACKNOWLEDGMENTS}

This work was supported by research Grant No. 15483 from VILLUM FONDEN and Enabling Research Grant No. ENRMFE19.DTU-03 from the EUROfusion Consortium. This work was carried out within the framework of the EUROfusion Consortium and received funding from the Euratom research and training program 2014-2018 and 2019-2020 under Grant Agreement No. 633053. The views and opinions expressed herein do not necessarily reflect those of the European Commission. This work was performed using resources provided by the ARCHER HPC through 
Grant No. EP/R029148/1, the Marconi HPC, as well as by the Cambridge Service for Data Driven Discovery (CSD3) operated by the University of Cambridge Research Computing Service (http:// www.csd3.cam.ac.uk/), provided by Dell EMC and Intel using Tier2 funding from the Engineering and Physical Sciences Research Council (capital Grant No. EP/P020259/1), and DiRAC funding from the Science and Technology Facilities Council (www.dirac.ac.uk).

\section{DATA AVAILABILITY}

The data that support the findings of this study are openly available in DTU Data at https://doi.org/10.11583/DTU.11847174 (Ref. 57).

\section{REFERENCES}

${ }^{\mathbf{1}}$ M. Faraday, Philos. Trans. R. Soc. London 121, 319 (1831).

${ }^{2}$ C. M. Topaz, J. Porter, and M. Silber, Phys. Rev. E 70, 066206 (2004).

${ }^{3}$ A. A. Sukhorukov, Y. S. Kivshar, O. Bang, and C. M. Soukoulis, Phys. Rev. E 63, 016615 (2000)

${ }^{4}$ V. P. Silin, Sov. Phys. JETP 21, 1127 (1965).

${ }^{5}$ Yu. M. Aliev, V. P. Silin, and C. Watson, Sov. Phys. JETP 23, 626 (1966).

${ }^{6}$ T. Amano and M. Okamoto, J. Phys. Soc. Jpn. 26, 529 (1969).

${ }^{7}$ A. D. Piliya, in Proceedings of the 10th International Conference on Phenomena in Ionized Gases, edited by R. N. Franklin (Donald Parsons and Co. Ltd., Oxford, 1971), p. 320.

${ }^{8}$ M. N. Rosenbluth, Phys. Rev. Lett. 29, 565 (1972)

${ }^{9}$ A. D. Piliya, Zh. Eksp. Teor. Fiz. Pis'ma Red. 17(7), 374-376.

${ }^{10}$ B. Thidé, H. Kopka, and P. Stubbe, Phys. Rev. Lett. 49, 1561-1564 (1982).

${ }^{11}$ A. Samimi, W. A. Scales, H. Fu, P. A. Bernhardt, S. J. Briczinski, and M. J. McCarrick, JGR: Space Phys. 118, 502-514 (2013).

${ }^{12}$ R. B. White and F. F. Chen, Plasma Phys. 16, 565-587 (1974).

${ }^{13}$ J. Preinhaelter and V. Kopecký, J. Plasma Phys. 10, 1 (1973).

${ }^{14}$ A. K. Ram and S. D. Schultz, Phys. Plasmas 7, 4084 (2000).

${ }^{15}$ G. Castro, D. Mascali, S. Gammino, G. Torrisi, F. P. Romano, L. Celona, C. Altana, C. Caliri, N. Gambino, D. Lanaia et al., Plasma Sources Sci. Technol. 26, 055019 (2017).

${ }^{16}$ V. K. Yadav and D. Bora, Phys. Plasmas 11, 4582 (2004).

${ }^{17}$ F. S. McDermott, G. Bekefi, K. E. Hackett, J. S. Levine, and M. Porkolab, Phys. Fluids 25, 1488 (1982).

${ }^{18}$ R. Wilhelm, V. Erckmann, G. Janzen, W. Kasparek, G. Müller, E. Räuchle, P. G. Schüller, K. Schwörer, and M. Thumm, Plasma Phys. Controlled Fusion 26, 1433 (1984).

${ }^{19}$ H. P. Laqua, Plasma Phys. Controlled Fusion 49, R1 (2007).

${ }^{20}$ D. G. Bulyginskii, V. V. Dyachenko, M. A. Irzak, M. M. Larionov, L. S. Levin, G. A. Serebrenyi, and N. V. Shustova, Sov. J. Plasma Phys. 12, 77-80 (1986).

${ }^{21}$ Z. A. Pietrzyk, A. Pochelon, R. Behn, A. Bondeson, M. Dutch, T. P. Goodman, M. Q. Tran, and D. R. Whaley, Nucl. Fusion 33, 197 (1993).

${ }^{22}$ V. Shevchenko, G. Cunningham, A. Gurchenko, E. Gusakov, B. Lloyd, M. O’Brien, A. Saveliev, A. Surkov, F. Volpe, and M. Walsh, Fusion Sci. Technol. 52(2), 202-215 (2007).

${ }^{23}$ A. Köhn, G. Birkenmeier, A. Chusov, P. Diez, A. Feuer, U. Höfel, H. Höhnle, E. Holzhauer, W. Kasparek, S. Merli et al., Plasmas Phys. Controlled Fusion 55, 014010 (2013).

${ }^{24}$ S. Kubo, M. Nishiura, K. Tanaka, D. Moseev, S. Ogasawara, T. Shimozuma, Y. Yoshimura, H. Igami, H. Takahashi, T. I. Tsujimura, and R. Makinoa, J. Instrum. 11, C06005 (2016).

${ }^{25}$ S. K. Nielsen, P. K. Michelsen, S. K. Hansen, S. B. Korsholm, F. Leipold, J. Rasmussen, M. Salewski, M. Schubert, M. Stejner, J. Stober et al., Phys. Scr. 92, 024001 (2017).

${ }^{26}$ S. K. Hansen, S. K. Nielsen, M. Salewski, M. Stejner, J. Stober, and ASDEX Upgrade Team, Plasma Phys. Controlled Fusion 59, 105006 (2017).
${ }^{27}$ S. K. Hansen, S. K. Nielsen, J. Stober, J. Rasmussen, M. Salewski, M. Stejner, and ASDEX Upgrade Team, Phys. Plasmas 26, 062102 (2019).

${ }^{28}$ M. Porkolab, V. Arunasalam, and N. C. Luhma, Plasma Phys. 17, 405 (1975)

${ }^{29}$ A. B. Altukhov, V. I. Arkhipenko, A. D. Gurchenko, E. Z. Gusakov, A. Yu. Popov, L. V. Simonchik, and M. S. Usachonak, EPL 126, 15002 (2019).

${ }^{30}$ V. K. Yadav and D. Bora, Phys. Plasmas 11, 3409 (2004).

${ }^{31}$ T. B. Leyser, Space Sci. Rev. 98, 223-328 (2001).

${ }^{32}$ T. D. Carozzi, B. Thidé, S. M. Grach, T. B. Leyser, M. Holz, G. P. Komrakov, V. L. Frolov, and E. N. Sergeev, J. Geophys. Res. 107(A9), 1253, https://doi.org/ 10.1029/2001JA005082 (2002).

${ }^{33}$ A. Najmi, B. Eliasson, X. Shao, G. M. Milikh, and K. Papadopoulos, Radio Sci. 51, 704-717, https://doi.org/10.1002/2015RS005866 (2016).

${ }^{34}$ D. C. Speirs, B. Eliasson, and L. K. S. Daldorff, J. Geophys. Res.: Space Phys. 122(10), 10638-10650, https://doi.org/10.1002/2017JA024665 (2017).

${ }^{35}$ N. E. Andreev, V. V. Pustovalov, V. P. Silin, and V. T. Tikhonchuk, ZhEFT Pis. Red. 18, 10 (1973).

${ }^{36}$ W. Seka, D. H. Edgell, J. F. Myatt, A. V. Maximov, R. W. Short, V. N. Goncharov, and H. A. Baldis, Phys. Plasmas 16, 052701 (2009).

${ }^{37}$ H. A. Baldis and C. J. Walsh, Phys. Rev. Lett. 47, 1658 (1981).

${ }^{38}$ A. G. Litvak, A. M. Sergeev, E. V. Suvorov, M. D. Tokman, and I. V. Khazanov, Phys. Fluids B 5, 4347 (1993).

${ }^{39}$ B. I. Cohen, R. H. Cohen, W. M. Nevins, and T. D. Rognlien, Rev. Mod. Phys. 63, 949 (1991)

${ }^{40}$ M. Porkolab and B. I. Cohen, Nucl. Fusion 28, 239 (1988).

${ }^{41}$ E. Z. Gusakov and A. Yu. Popov, Nucl. Fusion 51, 073028 (2011).

${ }^{42}$ E. Westerhof, S. K. Nielsen, J. W. Oosterbeek, M. Salewski, M. R. De Baar, W. A. Bongers, A. Bürger, B. A. Hennen, S. B. Korsholm, F. Leipold et al., Phys. Rev. Lett. 103, 125001 (2009).

${ }^{43}$ S. K. Nielsen, M. Salewski, E. Westerhof, W. Bongers, S. B. Korsholm, F. Leipold, J. W. Oosterbeek, D. Moseev, M. Stejner, and TEXTOR Team, Plasma Phys. Controlled Fusion 55, 115003 (2013).

${ }^{44}$ S. K. Nielsen, A. Jacobsen, S. K. Hansen, S. B. Korsholm, F. Leipold, J. Rasmussen, M. Salewski, M. Stejner, S. Denk, J. Stober et al., "Three-wave interaction during electron cyclotron resonance heating and current drive," in 41st International Conference on Infrared, Millimeter, and Terahertz Waves (IRMMW-THz), Copenhagen, Denmark, 25-30 September 2016 (IEEE, 2016).

${ }^{45} \mathrm{~S}$. K. Hansen, "Parametric decay instabilities in the electron cyclotron resonance heating beams at ASDEX upgrade," Ph.D. thesis (Technical University of Denmark, 2019).

${ }^{46}$ S. K. Hansen, S. K. Nielsen, J. Stober, J. Rasmussen, M. Stejner, M. Hoelzl, T. Jensen, and the ASDEX Upgrade Team, "Parametric decay instabilities near the second-harmonic upper hybrid resonance in fusion plasmas" (unpublished).

${ }^{47}$ E. Z. Gusakov and A. Yu. Popov, AIP Conf. Proc. 1187, 645 (2009).

${ }^{48}$ A. Yu. Popov and E. Z. Gusakov, J. Exp. Theor. Phys. 121(2), 362-374 (2015).

${ }^{49}$ A. Yu. Popov and E. Z. Gusakov, Plasma Phys. Controlled Fusion 57, 025022 (2015).

${ }^{50}$ E. Z. Gusakov and A. Yu. Popov, Phys. Plasmas 23, 082503 (2016).

${ }^{51}$ E. Z. Gusakov, A. Yu. Popov, and A. N. Saveliev, Phys. Plasmas 25, 062106 (2018).

${ }^{52}$ E. Z. Gusakov and A. Yu. Popov, Nucl. Fusion 59, 104003 (2019).

${ }^{53}$ T. D. Arber, K. Bennett, C. S. Brady, A. Lawrence-Douglas, M. G. Ramsay, N. J. Sircombe, P. Gillies, R. G. Evans, H. Schmitz, A. R. Bell, and C. P. Ridgers, "Contemporary particle-in-cell approach to laser-plasma modeling," Plasma Phys. Controlled Fusion 57, 113001 (2015).

${ }^{54}$ M. G. Senstius, S. K. Nielsen, R. G. Vann, and S. K. Hansen, Plasma Phys. Controlled Fusion 62, 025010 (2020).

${ }^{55}$ A. V. Arefiev, I. Y. Dodin, A. Köhn, E. J. Du Toit, E. Holzhauer, V. F. Shevchenko, and R. G. L. Vann, Nucl. Fusion 57, 116024 (2017).

${ }^{56}$ E. Z. Gusakov, A. Yu. Popov, and P. V. Tretinnikov, Nucl. Fusion 59, 106040 (2019).

${ }^{57}$ M. G. Senstius, S. K. Nielsen, and R. G. L. Vann (2020). "Numerical investigations of parametric decay into trapped waves in magnetized plasmas with a non-monotonic density background," DTU Data, https://doi.org/10.11583/ DTU.11847174 